\title{
ATENCIÓN TEMPRANA EN TIEMPOS DE COVID-19: INVESTIGAR LA/S REALIDAD/ES DE LA TELEINTERVENCIÓN EN LAS PRÁCTICAS CENTRADAS EN LA FAMILIA
}

\section{Early Childhood Intervention in Times of COVID-19: Exploring the Reality/ies of Tele-intervention in Family-Centred Practices (FCP)}

Lidia Rodríguez García

Universidad de Castilla-La Mancha. Facultad de Ciencias de la Salud. Departamento

de Pedagogía

Lidia.Rodriguez@uclm.es

Iván Herrán García

Plena inclusión Castilla-La Mancha

Paola de la Mano Rodero

Plena inclusión Castilla-La Mancha

Cristina DíAz SÁNCHez

ASPRONA Albacete

Juan Martínez CarRillo

ASPRONA Albacete

Recepción: 15 de octubre de 2020

Aceptación: 5 de febrero de 2021

RESUMEN: El equipo TRASCIENDE surge a partir de la necesidad de articular un proyecto de colaboración entre Plena inclusión-CLM y la UCLM para la transformación social, científica, académica y profesional en materia de discapacidad, inclusión y ciudadanía. A raíz de la situación sociosanitaria provocada por la COVID-19, el equipo TRASCIENDE se ha visto implicado en el cambio social que ha producido el confinamiento, desarrollando, necesariamente, un rol de observador del cambio. Esta situación global nos lleva a formularnos preguntas que pretenden iniciar procesos de investigación en la práctica: ¿de qué manera este confinamiento ha afectado a las prácticas en Atención Temprana? 


\section{ATENCIÓN TEMPRANA EN TIEMPOS DE COVID-I9: INVESTIGAR LA/S REALIDAD/ES DE LA TELEINTERVENCIÓN EN LAS PRÁCTICAS CENTRADAS EN LA FAMILIA L. RODRÍGUEZ, I. HERRÁN, P. DE LA MANO, C. DÍAZ Y J. MARTínEZ}

Para responder a esta pregunta se decide adoptar una perspectiva de investigación cualitativa para explorar las percepciones de familias y profesionales (logopedas, fisioterapeutas, estimuladoras y coordinadoras) de equipos de Atención Temprana, sus dificultades y sus fortalezas en esta situación de cambio. El trabajo describe un proceso de exploración de las prácticas durante los primeros meses del confinamiento, prácticas que se han dirigido, en muchos casos, hacia la teleintervención. Los datos han sido recogidos mediante entrevistas etnográficas (familias) y grupos focales (profesionales) y han sido tratados mediante análisis temático (Attride-Stirling, 2001). Los resultados muestran la percepción de las familias y los profesionales articulados en tres focos de redes temáticas: la reacción personal y colectiva a la nueva situación, lo que puede permanecer de la teleintervención (con sus matices) y lo que subyace a las prácticas en esta modalidad. El estudio abre, además, nuevas líneas de investigación futuras que evidencian la dificultad del proceso de transformación hacia modelos verdaderamente centrados en la familia.

Palabras clave: Atención Temprana; COVID-19; confinamiento; teleintervención; familias; profesionales; prácticas centradas en la familia (PCF).

AвSTRACT: TRASCIENDE, as a research team, arose from the need to articulate a collaborative project between Full Inclusion-CLM and the UCLM for social, scientific, academic, and professional transformation in the field of disability, inclusion, and citizenship. As a result of the socio-health situation provoked by COVID-19, the TRASCIENDE team has been involved in the social change produced by confinement, necessarily developing a role of observer of the change. This global situation leads us to ask ourselves questions that aim to initiate research processes in practice: how has this confinement affected practices in early care? To answer this question we decided to adopt a qualitative research perspective to explore the perceptions of families and professionals (speech therapists, physiotherapists, stimulators and coordinators) of Early Care teams, their difficulties and their strengths in this situation of change. The work describes a process of exploring practices during the first months of confinement, practices that have been directed, in many cases, towards tele-intervention. Data has been collected through ethnographic interviews (families) and focus groups (professionals) and has been processed through thematic analysis (Attride-Stirling, 2001). The results show the perception of families and professionals articulated in three thematic network focuses: the personal and collective reaction to the new situation, what can remain of the tele-intervention (with its nuances) and what underlies the practices in this modality. The study also opens new lines of future research which evidence the difficulty of the process of transformation towards actual family-centred models.

KeYwords: Early Childhood Intervention; COVID-19; confinement; tele-practice; families; professionals; family-centred practices (FCP).

\section{Introducción}

La luz viene a nosotros por la sensibilidad; sin sensibilidad visual no hay luz, no hay movimiento.

Robert Delaunay, Light (1912)

Ediciones Universidad de Salamanca / CC BY-NC-ND

Siglo Cero, vol. 52, número extraordinario, 2021, pp. 75-117

$$
-76-
$$


ATENCIÓN TEMPRANA EN TIEMPOS DE COVID-I 9: INVESTIGAR LA/S REALIDAD/ES DE LA TELEINTERVENCIÓN EN LAS PRÁCTICAS CENTRADAS EN LA FAMILIA L. RODRÍGUEZ, I. HERRÁN, P. DE LA MANO, C. DÍAZ Y J. MARTínEZ

\subsection{Presentación}

$\mathrm{E}$ 1 equipo TRASCIENDE surge a partir de la necesidad de articular un proyecto de colaboración entre Plena inclusión-CLM y la UCLM para la trasformación social, científica, académica y profesional en materia de discapacidad, inclusión y ciudadanía. El nacimiento de TRASCIENDE en 2017 se acompaña de un proceso de reflexión y análisis sobre la necesidad de que investigación (universidad) y práctica real (movimiento asociativo) comiencen a caminar juntas desde un marco de colaboración estable y planificado. Es momento de que el proceso de transformación en el que se encuentran inmersos gran número de servicios de Atención Temprana en España, Perú, México y Colombia traspase el ámbito de las organizaciones y pueda tener un reflejo en los diferentes títulos de grado formativos que se imparten en la Universidad, apoyando así el verdadero cambio cultural. De esta manera se contribuye, por un lado, a que el conocimiento científico proporcione bases para la práctica basada en la evidencia y, por otro, a que la investigación tenga una orientación aplicada.

\subsection{Contexto de transformación}

El proyecto nacional de transformación de los centros de Atención Temprana de Plena inclusión España "La Atención Temprana que queremos" ha tenido una especial repercusión en Castilla-La Mancha. Desde que el proyecto se puso en marcha en 2013 han sido muchos los centros que se han adherido a él, siendo actualmente 42, suponiendo el 78 \% de todos los centros de la región, estén asociados o no a Plena inclusión Castilla-La Mancha. El proyecto de transformación tiene como objetivo apoyar el camino de las entidades hacia un enfoque centrado en la familia, preferentemente en entorno natural. La Administración de Castilla-La Mancha, a través de la Consejería de Bienestar Social y su Dirección General de Discapacidad, ha estado desde el comienzo apoyando esta idea de transformación, tanto desde el punto de vista presupuestario como legislativo, siendo su plasmación el decreto por el que se regulan las condiciones mínimas exigibles a los centros y servicios destinados a la atención a personas con discapacidad en Castilla-La Mancha (Decreto 88/2017), donde se realiza una apuesta por el enfoque centrado en familia, prioritariamente en entorno natural.

\subsection{Contexto sociosanitario global del estudio}

La Organización Mundial de la Salud elevó el 11 de marzo de 2020 la situación de emergencia de salud pública ocasionada por la COVID-19 a pandemia internacional. En este contexto, el Gobierno de España aprueba el estado de alarma (Real Decreto 463/2020) el día 14 de marzo de 2020. Esta medida, recogida en la Constitución (artículo 116.2), habilita al Gobierno para tomar decisiones excepcionales en todo o en parte del territorio nacional, cuando se produzcan crisis sanitarias que supongan alteraciones graves de la normalidad. En este caso la decisión fue decretar el confinamiento 
ATENCIÓN TEMPRANA EN TIEMPOS DE COVID-I 9: INVESTIGAR LA/S REALIDAD/ES DE LA TELEINTERVENCIÓN EN LAS PRÁCTICAS CENTRADAS EN LA FAMILIA L. RODRÍGUEZ, I. HERRÁN, P. DE LA MANO, C. DÍAZ Y J. MARTíneZ

de toda la población del país, pudiendo salir de las casas solo en situaciones de gran necesidad. El estado de alarma, inicialmente de 15 días, fue prorrogándose hasta el 21 de junio de 2020. Por esta razón, todos los profesionales de los centros de Atención Temprana de Castilla-La Mancha tuvieron que realizar su labor desde su casa.

\subsection{La oportunidad para parar. La nueva realidad. Tantas realidades}

En marzo algo cambió e hizo cambiar todo. Académicos, profesionales, gestores, organizaciones y familias comenzamos a aprender a vivir en una nueva realidad. La crisis global que trajo el coronavirus y su enfermedad manifiesta, la COVID-19, forzó a parar y a transitar por un confinamiento individual y colectivo dirigido a estabilizar la salud comunitaria.

Esta nueva realidad (que no solo trae incertidumbre y miedos, sino que también dispara creatividades) ha permitido que el equipo TRASCIENDE reflexione, priorice y se replantee otras formas de acercamiento a la investigación-acción. Nuevas formas de trabajo que permitan, conforme al espíritu con el que nació el equipo, dar respuesta a la necesidad social, bienestar colectivo en esta nueva realidad, en estas tantas realidades.

\subsection{Vivir el cambio, observar el cambio e investigar el cambio a través de las narrativas}

Cada uno de los protagonistas de estas nuevas situaciones, de estas tantas realidades diversas, hemos vivenciado un cambio personal. Pero, además, nos corresponde realizar un cambio en las formas de desarrollar nuestras prácticas. El equipo TRASCIENDE se ha visto protagonista, como todos, de este cambio vital, pero, además, ha desarrollado un rol de observador del cambio. Este rol es el que nos lleva a formularnos preguntas que pretenden iniciar procesos de investigación en la práctica: ¿qué nuevas realidades surgen a raíz de un confinamiento?, ¿qué nuevas necesidades de apoyo se presentan?, ¿cómo se reorientan las prácticas en Atención Temprana?, ¿ de qué manera este confinamiento ha afectado a las prácticas en Atención Temprana? La investigación puede responder a estas preguntas desde diferentes perspectivas. Desde la perspectiva de investigación etnográfica que guía este estudio, los investigadores se acercan a la familia y los profesionales para conocer y comprender su percepción, su mundo, sus valores, sus creencias, sus interacciones, su comunicación, sus dificultades y sus fortalezas en esta situación de cambio. Para ello, la entrevista etnográfica (Westby, 1990), los grupos focales (Edmunds, 1999; Puchta y Potter, 2004; Juan y Roussos, 2010) y el análisis de redes temáticas (Attride-Stirling, 2001; Braun y Clarke, 2006) resultan especialmente útiles en esta propuesta. Los métodos cualitativos son más apropiados para explorar las experiencias de los padres (Aharony y Strasser, 1993) y las narrativas en grupo pueden ser una mejor manera de lograr respuestas más abiertas, reflexivas e informativas (Owens y Batchelor, 1996).

El análisis temático es un método de investigación cualitativa para identificar, analizar e informar de patrones/temas a partir de datos concretos con el objetivo de 


\section{ATENCIÓN TEMPRANA EN TIEMPOS DE COVID-I 9: INVESTIGAR LA/S REALIDAD/ES DE LA TELEINTERVENCIÓN EN LAS PRÁCTICAS CENTRADAS EN LA FAMILIA \\ L. RODRÍGUEZ, I. HERRÁN, P. DE LA MANO, C. DÍAZ Y J. MARTÍNEZ}

obtener una mayor comprensión de la realidad que se estudia. La técnica de las redes temáticas es una herramienta sensible para la sistematización y presentación de los análisis. Los análisis temáticos tratan de desenterrar los temas destacados en un texto a diferentes niveles; las redes temáticas tienen por objeto facilitar la estructuración y la representación de estos temas y sistematizan la extracción de: (1) Temas básicos, de menor orden evidente en el texto; (2) Temas de organización, categorías de temas básicos agrupados juntos para resumir principios más abstractos; y (3) Temas globales, temas superordinados que encapsulan las principales metáforas del texto como un todo. Estos son representados como mapas de red que describen los temas más importantes en cada uno de los tres niveles e ilustran las relaciones entre ellos.

\subsection{Oportunidad de explorar la teleintervención}

Por todo lo expuesto, nos encontramos ante un momento histórico único con una oportunidad excepcional para reflexionar y evaluar nuestras prácticas en Atención Temprana en estos últimos meses. A raíz del confinamiento, las prácticas se han transformado, necesariamente, en teleintervención y resultaba de extremo interés valorar cómo se han llevado a cabo, tanto desde el punto de vista de las familias como desde el punto de vista de los profesionales. Pensamos que la telepráctica o teleintervención permite a cada familia comunicarse con más personas, conectarse a otras realidades diversas y, por lo tanto, ampliar las vías de la colaboración y las oportunidades de generar nuevas o más robustas redes de apoyo. Veamos cuánto.

\section{Revisión histórica de la Atención Temprana}

En la última década la Atención Temprana (AT) está cambiando en todo el territorio (García-Sánchez, 2020). Las bases conceptuales de este cambio se asientan inicialmente en una necesidad de transformación cultural que no es exclusiva del campo de la Atención Temprana o de la realidad de nuestro país: (1) vienen de la mano del conocimiento actual sobre el desarrollo infantil (Karmiloff-Smith, 2003; Sameroff, 2009; Shonkoff, 2010), que entiende el desarrollo como resultado de múltiples interacciones, considera a la familia como una de las influencias más importantes sobre el desarrollo de la primera infancia (Kiernan, 2013), el entorno y las actividades diarias como las principales oportunidades de aprendizaje de los niños y el ámbito hacia al que deberían dirigirse las intervenciones y apoyos (Dunst et al., 2001; McWilliam, 2000, 2010); (2) se nutren del constructo de Discapacidad desarrollado por la Asociación Americana para la Discapacidad Intelectual (2010) y la Clasificación Internacional de la OMS del Funcionamiento, de la Discapacidad y la Salud (CIF, 2001), que consideran la discapacidad como una circunstancia entre la persona y su entorno, poniendo en valor el impacto de los apoyos individualizados para la mejora de su funcionamiento; (3) se apoyan en el reconocimiento de los derechos las personas con discapacidad intelectual y del desarrollo (ONU, 2006) que, tradicionalmente, 


\section{ATENCIÓN TEMPRANA EN TIEMPOS DE COVID-I9: INVESTIGAR LA/S REALIDAD/ES DE LA TELEINTERVENCIÓN EN LAS PRÁCTICAS CENTRADAS EN LA FAMILIA \\ L. RODRÍGUEZ, I. HERRÁN, P. DE LA MANO, C. DÍAZ Y J. MARTÍNEZ}

han experimentado una clara vulneración vinculada especialmente con situaciones de exclusión, como afirman Navas et al. (2017); (4) buscan la capacitación y empoderamiento de las familias como proceso y la mejora en la Calidad de Vida como resultado (Turnbull et al., 2004). Esta visión devuelve a las familias el papel protagonista en el desarrollo de sus hijos y en la toma de decisiones, al considerarlas destinatarias de los servicios, socias y colaboradoras.

La transformación en el campo de la Atención Temprana desde un enfoque centrado en el niño y en sus déficits hacia un enfoque centrado en la familia lleva implícito considerar a esta como una unidad de atención, reconocer y apoyar las competencias y fortalezas de las familias, alentar y respetar el derecho de los padres a tomar decisiones, lo que en la práctica debería suponer la participación e implicación de la familia en todos los procesos que se llevan a cabo desde que una familia llega a Atención Temprana hasta que finaliza el apoyo.

Investigaciones recientes muestran que el enfoque de prácticas centradas en la familia (PCF), desarrolladas en entornos naturales, se va extendiendo en España, provocando los necesarios cambios en la organización y el desarrollo de la intervención (Dalmau-Montala et al., 2017; Escorcia et al., 2018; García-Grau et al., 2019; GarcíaSánchez, 2018; García-Sánchez et al., 2014; García-Sánchez et al., 2018; García-Sánchez, 2020), aunque todavía con un amplio camino por recorrer.

Una parte crítica de esta transformación pasa por entender por qué la intervención en el ámbito de la Atención Temprana ha de pasar de considerar al niño como el receptor clave de la intervención, a ver a la familia como destinataria de los servicios y apoyos (Dunst et al., 2019; Moore, 2012; Raver y Childress, 2015). Resulta necesario redefinir la Atención Temprana en términos de experiencias, oportunidades y apoyos para la participación significativa en entornos como el hogar y la comunidad (Dunst y Trivette, 2009; Moore, 2012). Pero aún resulta un mayor desafío poder conectar la evidencia científica con las prácticas. En los últimos años se han incrementado exponencialmente el número de publicaciones en el campo de la Atención Temprana y apenas se ha trabajado en desarrollar mecanismos que faciliten el acceso al conocimiento o se dirijan a explorar el campo de la práctica basada en la evidencia (Dunst et al., 2019).

En esta dirección, la División para la Infancia Temprana (DEC, por sus siglas en inglés, Division Early Childhood) publica un trabajo (DEC, 2014) de gran impacto práctico que recoge la evidencia más actualizada, el conocimiento y la experiencia en el campo de la primera infancia, organizada alrededor de 8 áreas temáticas (liderazgo, evaluación, instrucción, familia, colaboración) que de un modo transversal están impregnadas de un enfoque centrado en familia, y abarcan cuestiones como la capacitación familiar y la colaboración entre la familia y el profesional. Estas prácticas han sido avaladas por numerosos organismos científicos (ASHA, 2008a; DEC, 2014; EURLYAID-EAECI, 2019) y en ellas se subraya que el profesional debe acompañar a la familia y a aquellos miembros responsables del cuidado del niño (Dunst et al., 2019) de cara a mejorar los resultados del aprendizaje y promover el desarrollo de los niños pequeños, desde el nacimiento hasta los 5 años.

Lo descrito hasta aquí pone de manifiesto que las prácticas recomendadas en Atención Temprana dibujan un perfil profesional complejo. El profesional de AT que 
ATENCIÓN TEMPRANA EN TIEMPOS DE COVID-I9: INVESTIGAR LA/S REALIDAD/ES DE LA TELEINTERVENCIÓN EN LAS PRÁCTICAS CENTRADAS EN LA FAMILIA

L. RODRÍGUEZ, I. HERRÁN, P. DE LA MANO, C. DÍAZ Y J. MARTÍNEZ

quiera trabajar desde un enfoque avalado por la evidencia se enfrenta a diferentes desafíos. El primero, dar respuesta a las necesidades de apoyo que tiene una familia con un niño con retraso o compromiso en su desarrollo, como señalan García-Sánchez et al. (2020), citando a Hughes-Scholes y Gavidia-Payne (2016). El segundo, facilitar que el niño disponga de las mejores oportunidades para su desarrollo. Por último, debe conocer y manejar estrategias de acompañamiento familiar y capacitación del adulto (García-Sánchez et al., 2020) para asegurarse de mantener su motivación y ayudarles a aprender y a sentirse seguros de sus capacidades para contribuir al desarrollo del menor (Dunst et al., 2014).

\subsection{Los motivos de asumir el reto que plantea el enfoque centrado en la familia}

El enfoque de prácticas centradas en la familia (PCF) es una filosofía que refleja el reconocimiento de que la familia está en el centro del aprendizaje del niño y, por lo tanto, es parte integral del proceso de AT (Bruder, 2000).

Las prácticas centradas en la familia suelen estar asociadas con resultados positivos para padres y madres, o cuidadores principales y niños (Dunst et al., 2007). Además, cuando los profesionales de AT apoyan la interacción responsable entre padres e hijos, los niños tienen más éxito en el cumplimiento de los logros de su desarrollo (Mahoney et al., 2004).

La investigación sobre las características de las PCF (Trivette y Dunst, 2000; Espe-Sherwindt, 2008) indica que este enfoque requiere, necesariamente, dos tipos de prácticas diferentes: prácticas de creación de relaciones o prácticas relacionales y prácticas de participación e interacción o prácticas participativas. Las prácticas relacionales incluyen cuestiones como la escucha activa y reflexiva; la comunicación efectiva; empatía; creencias de los profesionales sobre los puntos fuertes de la familia, y sensibilidad de los profesionales a los valores y las prácticas familiares personales y culturales (Hill, 2001). Las prácticas participativas o de creación de capacidad incluyen el comportamiento de los profesionales, la participación de los padres y otros familiares miembros en la elección informada y la toma de decisiones, basándose en la familia de los padres y otros los puntos fuertes de los miembros, promoviendo la adquisición de nuevas habilidades y participación de los padres tanto en la obtención de los recursos y apoyos necesarios como en la provisión de sus niños pequeños en oportunidades de aprendizaje que mejoran el desarrollo (Dunst y Trivette, 2009; Marco-Arenas et al., 2020).

\subsection{Aprender de las narrativas de las familias}

Aunque existe limitada investigación que explore las experiencias de padres y madres o cuidadores principales en Atención Temprana (Lyons, O’Malley et al., 2010), el interés por los padres como miembros activos en el desarrollo de sus hijos ha aumentado drásticamente en investigaciones de los últimos años (Watts et al., 2009). 
Los padres juegan un papel esencial en las prácticas centradas en la familia como el agente principal de intervención con su hijo (Klatte et al., 2020), sin embargo, las madres aún siguen siendo las participantes más implicadas en los servicios de intervención temprana (Curtiss et al., 2019).

Si bien actualmente se reconoce ampliamente el papel de los padres en el proceso de intervención, hasta la fecha se han realizado escasos estudios sobre sus experiencias en materia de Atención Temprana. En uno de estos estudios, Glogowska y Campbell (2000) determinaron que era necesario el diálogo entre los padres y los profesionales, en particular en las primeras etapas de la intervención, en las que el reconocimiento de la perspectiva de los padres podía influir en la futura adopción de decisiones y acuerdos de colaboración.

A pesar de la importancia de la participación de los padres en las PCF, la forma en que los padres participan difiere considerablemente entre los programas. Mas et al. (2020) sostienen que, sorprendentemente, sabemos muy poco sobre la relación entre las prácticas centradas en la familia y la participación de los padres (Rhoades, 2017) y sabemos aún menos sobre qué tipos de prácticas centradas en la familia están asociadas con el uso de las prácticas de PCF por parte de los padres con sus hijos pequeños (Lee, 2015).

Aun reconociendo la limitación en investigación, existe un acuerdo en las diez dimensiones teóricas que demandan las familias, que pueden inferirse de la literatura consultada (Dunst et al., 2002; Dunst y Trivette, 1996; Escorcia et al., 2016): colaboración con la familia, inclusión de la familia en la planificación de objetivos, promoción de la participación familiar, búsqueda de fortalezas familiares, búsqueda de recursos y oportunidades de participación, ayuda a la familia a considerar soluciones para los objetivos propuestos, apoyo a las decisiones de la familia, trabajo flexible e individualizado, atención a las necesidades y prioridades de la familia y enfoque de trabajo positivo.

\section{Estado actual de la teleintervención en Atención Temprana}

Existe evidencia científica que justifica el uso de la teleintervención para ofrecer una Atención Temprana de calidad. Grogan-Johnson et al. (2013) sostienen que la teleintervención tiene el potencial de proporcionar un mayor acceso a los servicios de Atención Temprana para los niños con dificultades de comunicación y sus familias. Existen otros estudios comparados (McCarthy et al., 2012; Behl et al., 2017) que subrayan los beneficios de la Atención Temprana a través de la intervención, en comparación con las tradicionales visitas a domicilio.

Específicamente, sobre el enfoque centrado en la familia y/o en entorno natural, los trabajos de Blaiser et al. (2013) y McCarthy et al. (2020) comparan la participación de los cuidadores principales en la teleintervención y la intervención temprana centrada en la familia de manera presencial, no encontrando diferencias remarcables. Otro criterio de acercamiento a la literatura serían las poblaciones susceptibles de recibir apoyo en AT mediante la intervención, ofreciendo la literatura ejemplos concretos de 
investigaciones en ámbito rural (Olsen et al., 2016; Cason, 2009). En relación con la prestación de servicios de Atención Temprana de alta calidad en el hogar y en la comunidad para niños con discapacidad que viven en zonas rurales y fronterizas, Olsen et al. (2016) sostienen que es un gran desafío y, al mismo tiempo, su viabilidad es una gran apuesta. La distancia, el clima, el terreno geográfico y la escasez de especialistas son los grandes desafíos para asegurar un acceso a los servicios adecuado y equitativo. Edwards et al. (2012) sostienen que hay un apoyo creciente de organizaciones profesionales para el uso de este modelo de prestación de servicios, como la tecnología de videoconferencia, que puede aliviar los efectos de la distancia en el acceso a la atención médica, si bien la literatura existente obliga a los profesionales a realizar más estudios relacionados específicamente con la efectividad y la eficacia de la telepráctica en Atención Temprana.

En relación con la muestra de los estudios, parece haber abundante evidencia sobre el uso de la teleintervención en niños con discapacidad auditiva de Behl y Houston (2012), siempre que se trate de un mecanismo de apoyo, no sustitutorio de la práctica en persona. Boisvert et al. (2010) realizan una revisión sistemática sobre teleintervención en la prestación de servicios de Atención Temprana con niños con trastorno del espectro autista. Estas autoras concluyen que la teleintervención es un prometedor enfoque de prestación de servicios en el tratamiento de niños con TEA. Un estudio reciente de Blake (2020) se centra en la mejora de la inteligibilidad del habla mediante la teleintervención en situación de pandemia sanitaria por la crisis de la COVID-19. Si bien no está dirigido a población en edad de AT, sostiene que la TI parece un modo apropiado de prestación de servicios que facilitó una práctica óptima de alta calidad y un servicio continuo a la mejora de la inteligibilidad del habla de las personas participantes durante el confinamiento.

Por último, señalar que el trabajo de Houston y Stredler-Brown (2012) afirma que la opción de la teleintervención garantiza que todas las familias reciben la intervención a la que tienen derecho si no hay otra alternativa.

La Tabla 1 recoge un resumen de los estudios categorizados por los focos de atención de los mismos. 


\begin{tabular}{|c|c|c|}
\hline \multicolumn{3}{|c|}{$\begin{array}{l}\text { TABLA 1. Evidencias en la investigación } \\
\text { sobre teleintervención en Atención Temprana }\end{array}$} \\
\hline Focos & Estudios & Conclusiones \\
\hline $\begin{array}{l}\text { Teleintervención } \\
\text { en Atención } \\
\text { Temprana }\end{array}$ & $\begin{array}{l}\text { McCarthy, Duncan y Leigh (2012); } \\
\text { Behl, Blaiser, Cook, Barrett, Callow- } \\
\text { Heusser, Brooks, Dawson, Quigley } \\
\text { y White (2017); McCarthy, Leigh } \\
\text { y Arthur-Kelly (2020); McCarthy, } \\
\text { Duncan y Leigh (2012); Behl, Blai- } \\
\text { ser, Cook, Barrett, Callow-Heusser, } \\
\text { Brooks, Dawson, Quigley y White } \\
\text { (2017); Molini-Avejonas, Rondon- } \\
\text { Melo, Albuquerque y Samelli (2015). }\end{array}$ & $\begin{array}{l}\text { La TI proporciona apoyos de calidad } \\
\text { en Atención Temprana. }\end{array}$ \\
\hline $\begin{array}{l}\text { Práctica } \\
\text { centrada en la } \\
\text { familia y/o } \\
\text { entorno natural }\end{array}$ & $\begin{array}{l}\text { Blaiser, Behl, Callow-Heusser y } \\
\text { White (2013); McCarthy, Leigh y } \\
\text { Arthur-Kelly (2020). }\end{array}$ & $\begin{array}{l}\text { Compara la participación de los cuida- } \\
\text { dores principales en la teleintervención } \\
\text { y en la intervención temprana centrada } \\
\text { en la familia de manera presencial, sin } \\
\text { encontrar diferencias notables. }\end{array}$ \\
\hline $\begin{array}{l}\text { Investigación } \\
\text { cualitativa }\end{array}$ & $\begin{array}{l}\text { Little, Kamholz, Corwin, Barrero- } \\
\text { Castillero y Wang (2016); Cason } \\
\text { (2009). }\end{array}$ & $\begin{array}{l}\text { Mediante el diseño de grupos de discu- } \\
\text { sión con familias y entrevistas a infor- } \\
\text { mantes clave utiliza el análisis de redes } \\
\text { temáticas. Resultados positivos en la te- } \\
\text { leintervención. }\end{array}$ \\
\hline $\begin{array}{l}\text { Cualificación } \\
\text { de } \\
\text { profesionales }\end{array}$ & $\begin{array}{l}\text { Houston y Stredler-Brown (2012), } \\
\text { Behl y Houston (2012), Grogan- } \\
\text { Johnson, Meehan, McCormick y } \\
\text { Miller (2015). }\end{array}$ & $\begin{array}{l}\text { Los profesionales no están cualificados } \\
\text { para llevar a cabo programas de Atención } \\
\text { Temprana utilizando la teleintervención } \\
\text { para apoyar verdaderamente a las familias. }\end{array}$ \\
\hline $\begin{array}{l}\text { Revisión siste- } \\
\text { mática en TI }\end{array}$ & $\begin{array}{l}\text { Molini-Avejonas, Rondon-Melo, } \\
\text { Albuquerque y Samelli (2015). }\end{array}$ & $\begin{array}{l}\text { El procedimiento de teleintervención } \\
\text { tenía ventajas sobre el enfoque alterna- } \\
\text { tivo de no teleintervención. }\end{array}$ \\
\hline \multirow{3}{*}{$\begin{array}{l}\text { Población } \\
\text { susceptible } \\
\text { de } \\
\text { teleintervención }\end{array}$} & $\begin{array}{l}\text { Discapacidad Auditiva: Olsen, Fie- } \\
\text { chtl y Rule (2016); Cason (2009). }\end{array}$ & $\begin{array}{l}\text { Discapacidad auditiva: la TI es un gran } \\
\text { desafío y al mismo tiempo su viabili- } \\
\text { dad es un gran reto. }\end{array}$ \\
\hline & Behl y Houston (2012). & \multirow{2}{*}{$\begin{array}{l}\text { La teleintervención es un prometedor } \\
\text { enfoque de prestación de servicios en } \\
\text { el tratamiento de los niños con TEA. }\end{array}$} \\
\hline & $\begin{array}{l}\text { TEA: Boisvert, Lang, Andriano- } \\
\text { poulos y Boscardin (2010). }\end{array}$ & \\
\hline Organizaciones & $\begin{array}{l}\text { Edwards, Stredler-Brown y Hous- } \\
\text { ton (2013). }\end{array}$ & $\begin{array}{l}\text { Creciente apoyo de las organizaciones } \\
\text { profesionales para el uso de este modelo } \\
\text { de prestación de servicios. }\end{array}$ \\
\hline $\begin{array}{l}\text { Teleinter- } \\
\text { vención en } \\
\text { COVID-19 }\end{array}$ & Blake (2020). & $\begin{array}{l}\text { La teleintervención ofrece buenos resul- } \\
\text { tados en la mejora de la inteligibilidad } \\
\text { del habla en bilingües en una situación } \\
\text { de pandemia por el COVID-19. }\end{array}$ \\
\hline $\begin{array}{l}\text { Garantizar dere- } \\
\text { chos y calidad de } \\
\text { la intervención }\end{array}$ & Houston y Stredler-Brown (2012). & $\begin{array}{l}\text { La teleintervención garantiza que todas las } \\
\text { familias reciban la intervención a la que tie- } \\
\text { nen derecho si no hay otra alternativa. }\end{array}$ \\
\hline
\end{tabular}




\subsection{Las Prácticas en Atención Temprana en tiempos de crisis por la COVID-19}

La incertidumbre frente al cuidado y el desarrollo de los niños pequeños puede afectar el funcionamiento de la familia, lo que genera estrés familiar (Dulguerián, 2012; Escartí et al., 2016; Frugone et al., 2020; González, 2009; Gray et al., 2011. En esta línea, Heimdahl y Serrano (2020) señalan una cuestión clave, ¿qué tipo de intervención es necesaria para que la dignidad del niño y la familia se preserven? La interrelación entre la disponibilidad y la calidad de los servicios, así como los recursos y capacidades familiares, inciden en las repercusiones en el desarrollo (DEC Practices, 2018; Mansilla et al., 2014).

Como señala García-Sánchez (2020), en un editorial reciente: “La situación vivida de confinamiento por los efectos de la pandemia de la COVID-19 ha supuesto un revulsivo importante en el campo de la Atención Temprana. Todos los profesionales se han visto en la necesidad de centrarse en la familia, ya que no podían actuar directamente con el niño. Con ello, se han visto obligados a atender justo uno de los principios fundamentales de las prácticas centradas en la familia” (p. 96).

\section{Objetivos del estudio}

El principal objetivo de este estudio era explorar la/s realidad/es de la Atención Temprana durante el confinamiento, tanto desde el punto de vista de las familias como de los profesionales. Para ello se establecen diferentes objetivos específicos: (1) explorar la percepción de las familias ante la atención recibida mediante la teleintervención; (2) explorar la percepción de los profesionales ante la atención ofrecida mediante la teleintervención; (3) explorar la percepción de los coordinadores de centros ante la atención ofrecida mediante la teleintervención; (4) conocer en profundidad barreras y facilitadores de este medio de apoyo en Atención Temprana, y (5) valorar la incorporación de la teleintervención como un medio más en las prácticas de Atención Temprana tras la crisis de la COVID-19.

\section{Método}

\subsection{Diseño}

Desde una aproximación cualitativa, se llevan a cabo entrevistas etnográficas (Westby, 1990) con las familias seleccionadas. El término "conversación con un formato semiestructurado" (Guthrie y Stansfield, 2015) se acerca más al desarrollo real de las entrevistas realizadas, destacando el clima de confianza y cordialidad entre las familias y el equipo de investigación. Se trata, en definitiva, de escuchar cómo la persona ha sentido, percibido o experimentado lo que ha ocurrido (Westby, 1990), siendo esto último, precisamente, el objetivo de este estudio. 
ATENCIÓN TEMPRANA EN TIEMPOS DE COVID-I9: INVESTIGAR LA/S REALIDAD/ES DE LA TELEINTERVENCIÓN EN LAS PRÁCTICAS CENTRADAS EN LA FAMILIA L. RODRÍGUEZ, I. HERRÁN, P. DE LA MANO, C. DÍAZ Y J. MARTínEZ

\subsection{Cuestiones éticas}

Todos los participantes en el estudio cumplimentaron un documento de consentimiento informado a través de un formulario en google forms en el que aceptaban su participación y la cesión de datos con carácter investigador, bajo custodia de Plena inclusión Castilla-La Mancha.

\subsection{Participantes}

En este estudio participan voluntariamente y bajo asignación aleatoria tanto familias como profesionales de Atención Temprana de las cinco provincias de la Comunidad Autónoma de Castilla-La Mancha. El equipo TRASCIENDE realizó una convocatoria inicial a los centros y servicios de AT que forman parte del proyecto de transformación de AT de Plena inclusión, en Castilla-La Mancha. El número corresponde al $78 \%$ de los centros/servicios de AT públicos y concertados de CLM para que informaran sobre el estudio a profesionales y familias, así como la posibilidad de ser seleccionados. Posteriormente los centros informaron a los participantes de la muestra final, obtenida de manera aleatoria. TRASCIENDE contactó, ya directamente, con los participantes seleccionados con el fin de acordar las fechas pertinentes para los encuentros. Se realizaron, por una parte, entrevistas etnográficas semiestructuradas a 12 familias y, por otra, cuatro grupos focales con 32 profesionales de 22 centros/servicios de AT de CLM, divididos en base a las especialidades reflejadas en el Decreto 88/2017, de 5 de diciembre, por el que se regulan las condiciones mínimas exigibles a los centros y servicios destinados a la atención a personas con discapacidad en Castilla-La Mancha.

\subsection{Procedimiento}

Las entrevistas etnográficas se llevaron a cabo durante la primera semana de julio de 2020, si bien algunas familias tuvieron que cambiar las fechas iniciales confirmadas por cuestiones personales. El equipo se adaptó con flexibilidad a todos los cambios, así como a la variabilidad de los tiempos de duración.

Los grupos focales se programaron durante la última semana de junio de 2020 y tuvieron una duración entre 90 y 100 minutos. Se llevó a cabo un grupo focal online cada mañana, siguiendo este orden: logopedas, fisioterapeutas, estimuladoras y, por último, coordinadoras de equipos, de acuerdo con los perfiles señalados anteriormente.

\subsection{Análisis de datos}

Las entrevistas se realizaron online a través de la plataforma Zoom, o telefónicamente en un caso en el que no fue posible la conexión, por una o varias personas del 
equipo. Los registros en audio y vídeo (o ambos, en su mayoría) se transcribieron verbatim con el programa profesional sonix, en su versión de pago y bajo custodia única del equipo en un ordenador con contraseña protegida, y se editaron y depuraron posteriormente. Los registros y transcripciones fueron completados con notas pre-, peri- y postentrevistas/grupos, tomadas por el equipo durante el proceso de cara a incrementar la transparencia descriptiva e interpretativa (Maxwell, 2008).

Los datos fueron analizados individualmente y luego de manera colectiva por el equipo siguiendo el análisis de redes temáticas (Attride-Stirling, 2001). El proceso fue compartido y debatido en todo momento. Se utilizó, siguiendo a Curtiss et al. (2019), un enfoque de colaboración para la codificación a través de proceso reflexivo compartido y controlando las suposiciones y el sesgo de los investigadores. Attride-Stirling (2001) describe seis etapas en el procedimiento de análisis temático. Como el objetivo del estudio era explorar la/s realidad/es de las familias y profesionales en Atención Temprana durante el confinamiento, para la primera etapa se estableció un código marco para determinar cuestiones contextuales descritas en las entrevistas y grupos focales. En segundo lugar, se categorizan cuestiones detalladas por los participantes en las que manifiestan especial énfasis mediante repeticiones, lenguaje no verbal y nivel de acuerdo o asentimiento, entre otras. Las etapas siguientes se desplazan desde los códigos a los temas propiamente e identifican y refinan la interpretación del texto en temas discretos, pero amplios y tácitos (Attride-Stirling, 2001), conforme al acuerdo previo del equipo para este trabajo. Las redes temáticas, los temas y las categorías que se desprenden del análisis fueron discutidas y consensuadas por el equipo investigador en diferentes sesiones de trabajo.

Los datos del análisis preliminar fueron discutidos tanto entre el equipo interno como con el equipo de investigación en Thematic Network Analysis, de la Manchester Metropolitan University, en una reunión online. Esto permitió la exploración de transferibilidad y la interpretación de temas con la suficiente credibilidad y rigor (Guba y Lincoln, 1994).

\section{Resultados}

Tras el análisis de los datos se crearon tres redes temáticas principales. Los tres temas globales (Global Themes, Atrride-Stirling, 2001) a partir de los cuales se establece la relación en red (Figura 1) fueron: Reacciono, Se queda -pero- y Lo que subyace, descritos en la Figura 1. 
ATENCIÓN TEMPRANA EN TIEMPOS DE COVID-I 9: INVESTIGAR LA/S REALIDAD/ES DE LA TELEINTERVENCIÓN EN LAS PRÁCTICAS CENTRADAS EN LA FAMILIA L. RODRÍGUEZ, I. HERRÁN, P. DE LA MANO, C. DÍAZ Y J. MARTÍNEZ

FiguRa 1. Temas globales, relación temática en red y categorías en este estudio

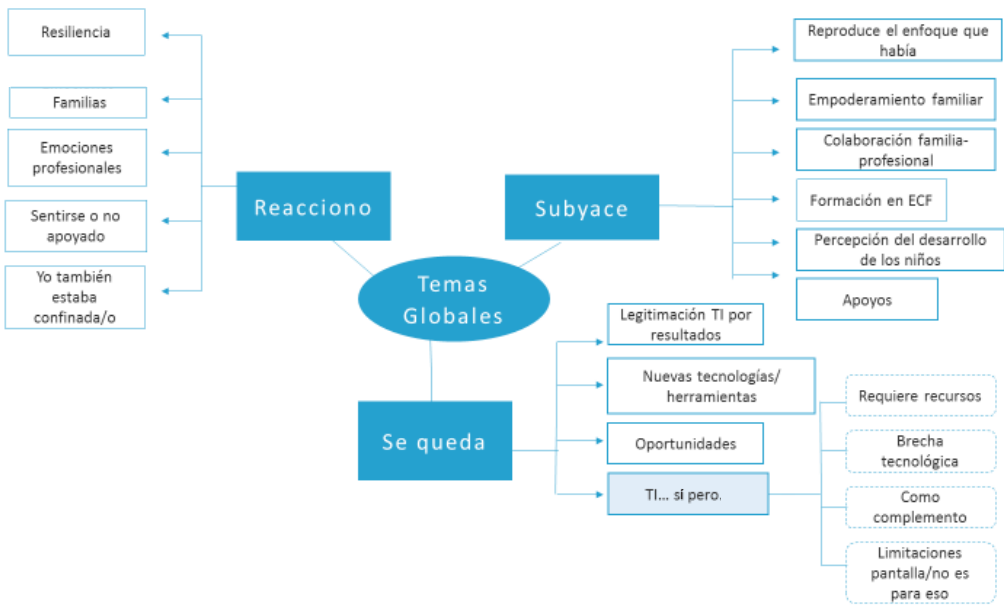

Las categorías que corresponden a cada tema global están también descritas en la Tabla 2. En el tema global se engloban una serie de categorías que se presentan al modo de otros estudios como Lyons et al. (2008, 2018), Klatte et al. (2019), Curtis et al. (2019), Kiernan (2019), Guthrie y Stansfield (2017) o Stansfield (2020). 


\begin{tabular}{|c|c|c|}
\hline \multicolumn{3}{|c|}{$\begin{array}{l}\text { TABLA 2. Redes temáticas analizadas: tema global, } \\
\text { descripción y categorías correspondientes }\end{array}$} \\
\hline Red/Tema & Descripción & Categorías que engloba \\
\hline $\begin{array}{l}\text { Red Temá- } \\
\text { tica } 1 . \\
\text { Tema } \\
\text { global: } \\
\text { Reacciono }\end{array}$ & $\begin{array}{l}\text { Esta red temática ofrece una mira- } \\
\text { da sobre la narrativa de las personas } \\
\text { que han participado en el estudio } \\
\text { (familias, profesionales y coordina- } \\
\text { doras) en el marco de una situación } \\
\text { de crisis sanitaria sobrevenida que } \\
\text { ha provocado diferentes impactos/ } \\
\text { reacciones: emocional, organizati- } \\
\text { vo, tecnológico, entre otros. }\end{array}$ & $\begin{array}{l}\text { Emociones profesionales } \\
\text { Emociones familias } \\
\text { Resiliencia } \\
\text { Sentirse apoyado o no } \\
\text { Yo también estaba confinada }\end{array}$ \\
\hline \multirow[t]{2}{*}{$\begin{array}{l}\text { Red Temá- } \\
\text { tica } 2 . \\
\text { Tema } \\
\text { global: } \\
\text { Se queda }\end{array}$} & $\begin{array}{l}\text { Esta categoría recoge la opinión } \\
\text { general de las familias y profesio- } \\
\text { nales en relación con apostar o no } \\
\text { por la TI como instrumento al ser- } \\
\text { vicio de las Prácticas de Atención } \\
\text { Temprana en entorno natural. }\end{array}$ & $\begin{array}{l}\text { Legitimación TI por resultados } \\
\text { Nuevas tecnologías/ } \\
\text { herramientas } \\
\text { Oportunidades } \\
\text { TI como Complemento }\end{array}$ \\
\hline & $\begin{array}{l}\text { Sí, pero. Este hilo engloba aquellas } \\
\text { opiniones defamilias yprofesionales } \\
\text { que se muestran a favor de la TI, } \\
\text { pero que añaden algún tipo de } \\
\text { condicionante. }\end{array}$ & $\begin{array}{l}\text { Brecha tecnológica } \\
\text { Limitaciones pantalla/no para esto } \\
\text { Requiere recursos/procesos } \\
\text { específicos } \\
\text { TI como complemento } \\
\end{array}$ \\
\hline $\begin{array}{l}\text { Red Temá- } \\
\text { tica } 3 . \\
\text { Tema } \\
\text { global: } \\
\text { Lo que } \\
\text { subyace }\end{array}$ & $\begin{array}{l}\text { Este tema recoge cómo las prácticas } \\
\text { durante el confinamiento han reve } \\
\text { lado distintos niveles de aproxima } \\
\text { ción al enfoque centrado en la fami- } \\
\text { lia, con implicaciones en la concep } \\
\text { ción sobre el rol profesional, la or- } \\
\text { ganización de los equipos y la fun- } \\
\text { cionalidad de lasintervenciones. }\end{array}$ & $\begin{array}{l}\text { Empoderamiento familiar } \\
\text { Colaboración familia-profesional } \\
\text { Formación ECF } \\
\text { Percepción del desarrollo de los } \\
\text { niños } \\
\text { Apoyos } \\
\text { La TI ha intentado reproducir el } \\
\text { enfoque que había }\end{array}$ \\
\hline
\end{tabular}

\section{Red temática 1. Tema global: Reacciono ante el confinamiento}

Las reacciones ante el confinamiento parecen tener un eje común tanto en familias como en profesionales, con base en su situación personal unida a la profesional y/o familiar particular. Aparece un fuerte componente emocional y la importancia de sentirse apoyadas por los profesionales (en el caso de las familias) y por los respectivos equipos (en el caso de los profesionales). Se destaca la capacidad de respuesta y adaptación a la nueva situación. Las categorías en esta red temática se ilustran en la Figura 2. 
Figura 2. Categorías en la red temática 1: Reacciono

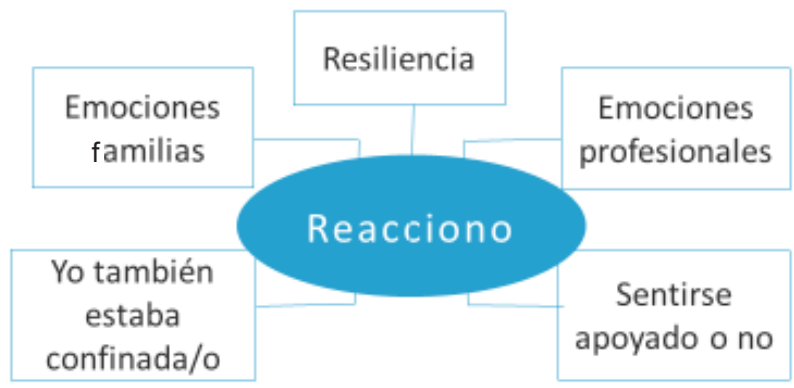

Entre las categorías analizadas en la red temática Reacciono, encontramos las expuestas en la Tabla 3, junto a algunos extractos de las narrativas analizadas. Entre las emociones de las familias destacan las vinculadas a cada situación familiar particular, estrés/sobrecarga y gratitud hacia los profesionales. Emociones de los profesionales, como agotamiento o incertidumbre. En el Sentirse apoyado: importancia de sentirse cuidados, tanto las familias como los profesionales. Resiliencia: entendida en este caso como la capacidad de mantener la colaboración y los apoyos a pesar de las dificultades. Yo también estaba confinada: el confinamiento de los propios profesionales como condicionante de la propia TI.

\begin{tabular}{|l|l|}
\hline \multicolumn{2}{|c|}{ TABLa 3. Categorías analizadas y extractos en la red temática 1: Reacciono } \\
\hline \multicolumn{1}{|c|}{ Categorías } & \multicolumn{1}{c|}{ Extractos de las narrativas } \\
\hline $\begin{array}{l}\text { Emociones } \\
\text { familias }\end{array}$ & $\begin{array}{l}\text { Lo siento mucho, pero es que no puedo, no puedo. Saco cinco minu- } \\
\text { tos para hablar contigo por teléfono, pero estoy fatal, en el trabajo, } \\
\text { estoy fatal personalmente (la profesional relata que algunas familias } \\
\text { han verbalizado estas cuestiones). } \\
\text { Y como llegó así este parón, a mí me daba mucho bajón. Muchas } \\
\text { veces le decía a mi marido, jolines, con lo bien que iba, que se habían } \\
\text { alineado todas las estrellas, todos trabajando en la misma dirección } \\
\text { y ha llegado esto. }\end{array}$ \\
\hline $\begin{array}{l}\text { Emociones } \\
\text { profesionales }\end{array}$ & $\begin{array}{l}\text { Ansiedad, al principio es un poco. De mucho estrés. No saber por } \\
\text { dónde vas a tirar, no sabes hacer las cosas. La familia tampoco sabe } \\
\text { qué tiene que hacer, ni qué le vas a pedir, ni nada. } \\
\text { Había que compaginar tu estrés personal de tu vida con tus cosas, } \\
\text { con el de las familias, pero en general, como todo el mundo estaba } \\
\text { igual, creo que todo el mundo era tolerante. } \\
\text { Incertidumbre: qué hacer, cuánto tiempo. Qué hacer con las familias } \\
\text { que acaban de empezar. }\end{array}$ \\
\hline
\end{tabular}

Ediciones Universidad de Salamanca / CC BY-NC-ND

Siglo Cero, vol. 52, número extraordinario, 2021, pp. 75-117 


\begin{tabular}{|l|l|}
\hline \multicolumn{2}{|l|}{ TABLA 3. Categorías analizadas y extractos en la red temática 1: Reacciono (cont.) } \\
\hline Categorías & \multicolumn{1}{c|}{ Extractos de las narrativas } \\
\hline $\begin{array}{l}\text { Sentirse } \\
\text { apoyado }\end{array}$ & $\begin{array}{l}\text { Muy bueno, como te decía en cualquier momento, daba igual que } \\
\text { fuera de diario que fue, ya que fue la sesión, que fuera un domingo } \\
\text { que Maca había hecho algo y yo la mandaba un vídeo. En cualquier } \\
\text { momento la puedo llamar o la podemos escribir. Y está dispuesta. } \\
\text { Si algo lo ha salvado, han sido los equipos. }\end{array}$ \\
\hline Resiliencia & $\begin{array}{l}\text { Sobre todo, resaltar la capacidad de adaptación, capacidad de actua- } \\
\text { ción, tanto de las familias, las primeras, del equipo. Nos confinaron } \\
\text { un viernes a nosotras y el lunes manos a la obra, dando lo mejor. }\end{array}$ \\
$\begin{array}{l}\text { En un viernes decidimos que no volvíamos y nos organizamos } \\
\text { para el lunes estar siguiendo con la agenda, tal y como la teníamos } \\
\text { planificada. }\end{array}$ \\
\hline $\begin{array}{l}\text { Yo } \\
\text { también } \\
\text { estaba } \\
\text { confinado/a }\end{array}$ & $\begin{array}{l}\text { Influye mucho el tipo de factores, si tienes hijos o no, si aparte tu } \\
\text { marido tenía que trabajar, si podía estar en casa [...] porque yo puedo } \\
\text { contar algo que yo he tenido el 100 por cien de mi jornada para de- } \\
\text { dicarme a las familias, pero sí que tengo compañeras que les ha sido } \\
\text { supercomplicado. } \\
\text { Pero este confinamiento he estado de noches. He trabajado de noches. } \\
\text { Entonces, por la noche los niños estaban durmiendo y no se entera- } \\
\text { ban de que no estaba. Y por la mañana, yendo a las once y media, me } \\
\text { levantaba, dormía muy poquito, pero ya estaba con ellos, le ayudaba } \\
\text { a la nena en los deberes del cole. Nos poníamos con el nene a trabajar } \\
\text { cosas, a ponerlo en la manta que jugara, pues a enseñarle cosas. }\end{array}$ \\
\hline
\end{tabular}

\section{Red temática 2. Tema global: La teleintervención se queda}

Las valoraciones de familias y profesionales sugieren que la teleintervención "ha venido para quedarse". Las familias destacan cuestiones como la posibilidad de contactar con otras familias, las oportunidades para la participación de ambos padres y de otros miembros de la familia o la facilidad para encajar las vistas, entrevistas, etc., en su dinámica habitual. Mientras que los profesionales narran este tipo de razones: posibilidades de mejora de las prácticas a través de la tecnología, efectividad de la TI para la capacitación familiar o disponibilidad de alternativas cuando no es posible la intervención presencial.

Entre las categorías analizadas en este tema global encontramos las descritas en la Tabla 4 que se describen como Legitimación TI por resultados: impresión de resultados positivos en el niño, las familias han desarrollado nuevas competencias y adquirido nuevos recursos, los profesionales valoran esta metodología como positiva. Nuevas tecnologias/herramientas: se incorporan nuevas herramientas: uso de 
vídeos, videoconferencia, descubren nuevas maneras de conectarse. Oportunidades: ofrece oportunidades para los profesionales de formación online en tiempo real y conexión, oportunidades de la participación de la familia. Utilidades TI: amplía la posibilidad de respuesta ante niños enfermos, largas distancias, vacaciones o condiciones atmosféricas que impidan desplazamientos, entre otras.

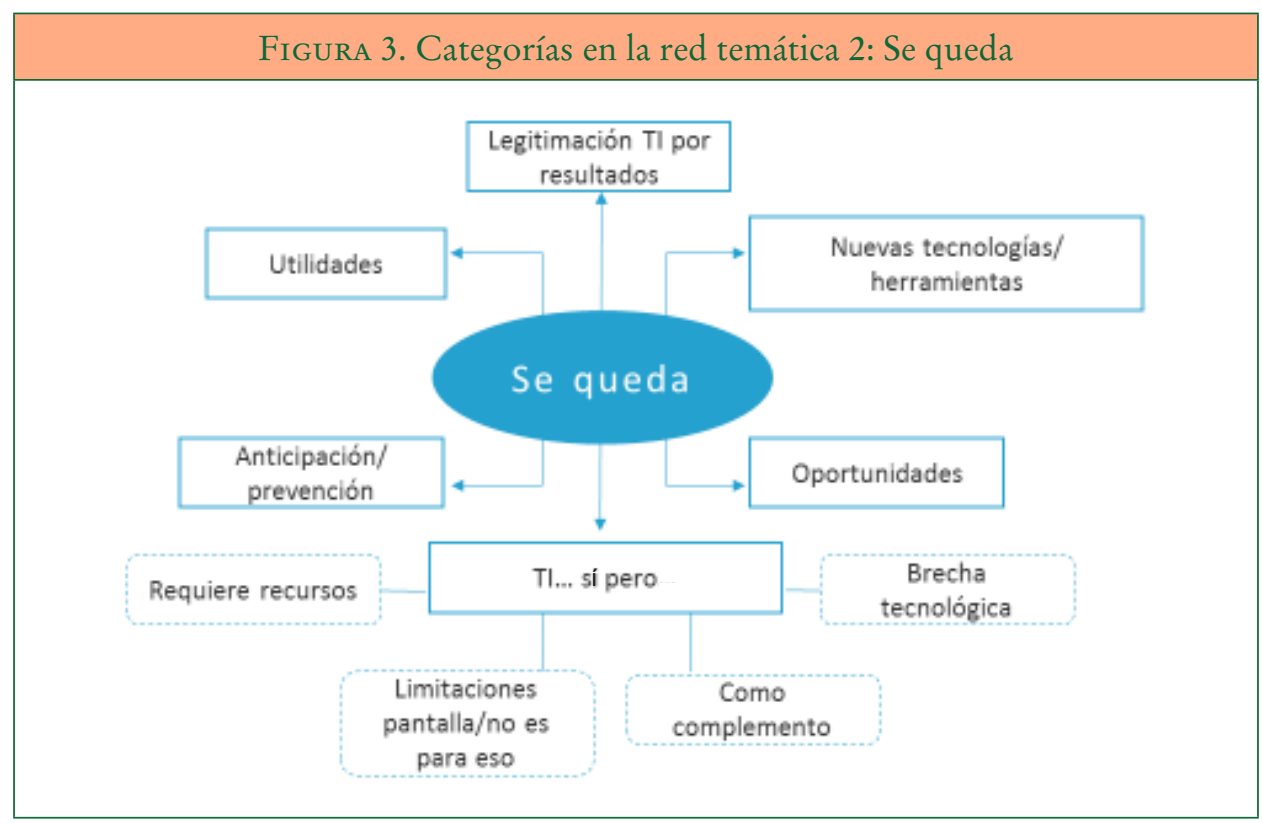

Así, se presentan en la Tabla 4 algunos extractos de narrativas ligados a estas categorías. 
ATENCIÓN TEMPRANA EN TIEMPOS DE COVID-I 9: INVESTIGAR LA/S REALIDAD/ES DE LA TELEINTERVENCIÓN EN LAS PRÁCTICAS CENTRADAS EN LA FAMILIA

L. RODRÍGUEZ, I. HERRÁN, P. DE LA MANO, C. DÍAZ Y J. MARTÍNEZ

TABLA 4. Categorías analizadas y extractos en la red temática 2: Se queda

Categorías

Legitimación TI por resultados

Oportunidades

Nuevas

tecnologías/

herramientas
Extractos de las narrativas

Cómo sin tocar podemos actuar. Cómo, sin estar ahí, también podemos llegar a las familias.

No, era igual. Igual. Es igual que si ella estuviera en mi casa, cualquier duda, ella siempre, es que siempre, siempre está pendiente, de verdad.

Hemos aprendido cosas que, aunque no sigamos con la teleintervención, se pueden llevar a la práctica: más uso de herramientas como vídeos y demás, que cuando ibas nos echaban en falta como ahora.

Nos comunicábamos a través de las videollamadas, a través de dúo, y luego por WhatsApp. Esas son las aplicaciones. Una vez utilizamos zoom, que fue cuando hicimos, porque nos pidieron, creo que te lo comenté, nos pidieron hacer un vídeo también sobre el confinamiento.

Al principio quería como atravesar la pantalla. Se me notaba cada vez más cerca del ordenador. Aquí, a través de cuanto más cerca estaba, mejor me van a entender y no era así. Entonces, al principio sentía como que necesitaba atravesar esa pantalla y estar ahí, y luego, poco a poco me di cuenta de que creo que me va a venir muy bien para cuando empecemos las presenciales, que no tengo que estar tan ahí.

Me he dado cuenta de que he tenido como una reconciliación, incluso con el modelo, porque yo era muy modelo sanitario, muy fisio, y eso también es un poco..., cuando me convertí en madre, mi visión cambia totalmente, pero a raíz de la teleintervención también, porque me he dado cuenta de que los padres están muy capacitados y que nosotros les tenemos que ayudar en este camino, pero no ser el papel principal de la evolución en el desarrollo de ese niño. Sí que he tenido una reconciliación enorme con el modelo.

Seguir haciéndolo así en mi caso concreto, además que me vendría fenomenal porque tenemos intención de trasladarnos a otra localidad y sería la manera perfecta para poder seguir atendiendo a mis hijos y que no tenga yo problemas de conciliación familiar. Con quién dejo a este para irme, para no irme. Que desde casa pudiera hacerlo.

Por otro lado, dentro del tema global "Se queda", en el que parece haber consenso entre las personas participantes, encontramos matices en relación con las condiciones en las que esta herramienta debe quedarse. En esta categoría, que matiza las condiciones en las que se debe quedar la teleintervención, encontramos las siguientes. Brecha tecnológica que supone necesidad de tener en cuenta que las dificultades de acceso a la red y de utilización de la tecnología han impedido la intervención con algunas familias. Limitaciones pantalla/no para esto: los nuevos sistemas de comunicación virtual 
no cubren el espectro de oportunidades no verbales de la relación cara a cara como la calidez emocional. Requiere recursos/procesos específicos: necesidad de utilizar recursos para la teleintervención que antes no existían (teléfonos móviles, ordenadores u otros). Teleintervención como complemento, no sustituye: es una fórmula que enriquece nuestras prácticas, cuenta con limitaciones.

\begin{tabular}{|c|c|}
\hline Categorías & Extractos de las narrativas \\
\hline $\begin{array}{l}\text { Requiere } \\
\text { recursos }\end{array}$ & $\begin{array}{l}\text { Y luego, por otro lado, los medios técnicos de los que disponemos, las } \\
\text { entidades. Nosotras hemos puesto todas nuestro teléfono personal a dis- } \\
\text { posición de las familias, que entendemos que sí, que es una situación de } \\
\text { emergencia y lo ha requerido así para poder seguir adelante con la inter- } \\
\text { vención. Pero es cierto que, como medida a largo plazo, deberíamos de } \\
\text { disponer de otros dispositivos para poderlo llevar o hacerla de calidad. } \\
\text { Medios tecnológicos, pues lo que cada uno tenía en casa; bueno yo cogía } \\
\text { un ordenador del centro. Tenemos un teléfono en el centro. Pero claro, } \\
\text { solo uno y el resto, pues estamos utilizando todos los que tenemos. }\end{array}$ \\
\hline $\begin{array}{l}\text { Brecha } \\
\text { tecnológica }\end{array}$ & $\begin{array}{l}\text { Nos dieron la opción porque entiendo que toda la familia no tiene } \\
\text { acceso a internet. No todas las familias tienen la opción, por lo que } \\
\text { le pregunté a Nieves que es mi profesional de referencia, pues ellos } \\
\text { han tenido la suerte de que todas las familias han podido acceder a } \\
\text { internet y demás, porque es que si no alguno se quedaría atrás. } \\
\text { Destacar que también el tema de las tecnologías, no todas las fami- } \\
\text { lias hemos podido contar con ellas, es el problema y luego también } \\
\text { la diversidad cultural. Es decir, hay familias que con el tema del } \\
\text { idioma también hemos tenido dificultades, hemos intentado buscar } \\
\text { estrategias donde poder apoyarnos y eso es un proceso de un traba- } \\
\text { jo que seguimos en ello, que es un poco complicado. }\end{array}$ \\
\hline $\begin{array}{l}\text { Como } \\
\text { complemento }\end{array}$ & $\begin{array}{l}\text { Vamos, sí o sí que ha llegado para quedarse, pero creo que la tene- } \\
\text { mos que incluir en nuestras rutinas de hacer. No sé con qué fre- } \\
\text { cuencia, pero la teleintervención puede ser una de las sesiones cada } \\
\text { equis tiempo y empoderar a las familias en este uso. } \\
\text { Así que creo que es una herramienta útil, pero no sustitutiva de la } \\
\text { intervención física. Desde mi punto de vista. } \\
\text { Yo veo que también es una herramienta muy necesaria para ser } \\
\text { complementaria en el futuro, con familias que lo necesiten por en- } \\
\text { fermedad, porque están lejos o por lo que sea. } \\
\text { Creo que el futuro no muy lejano irá hacia este método, no como mé- } \\
\text { todo único, sino como un método complementario a lo mejor para } \\
\text { ciertas familias que están lejos, bastante lejos de nuestro centro o inclu- } \\
\text { so a lo mejor para hacer seguimientos de niños que llevan ya meses en } \\
\text { mi intervención y veamos que van evolucionando positivamente. }\end{array}$ \\
\hline
\end{tabular}




\begin{tabular}{|l|l|}
\hline \multicolumn{2}{|c|}{ TABla 5. Categorías analizadas y extractos en la red temática 2: Sí, pero (cont.) } \\
\hline \multicolumn{1}{|c|}{ Categorías } & \multicolumn{1}{c|}{ Extractos de las narrativas } \\
\hline $\begin{array}{l}\text { Limitaciones } \\
\text { pantalla/no es } \\
\text { para eso }\end{array}$ & $\begin{array}{l}\text { [...] para eso me parece que es algo que es positivo y es una herra- } \\
\text { mienta útil, pero yo tengo muchas ganas de volver a las casas por- } \\
\text { que en algunas situaciones más complejas me he encontrado en este } \\
\text { tiempo, como un diagnóstico, momentos emocionales muy com- } \\
\text { plejos a través de la pantalla, me ha resultado más difícil acompañar } \\
\text { a esas familias que cuando me he encontrado con ellos en el entorno. } \\
\text { Creo que tiene más aspectos positivos que negativos, aunque creo } \\
\text { que cuando llegaba una nueva familia, o niño al centro, se hace la } \\
\text { entrevista y demás. Creo que es importante los primeros meses, di- } \\
\text { ría yo, crear ese vínculo con la familia, con el niño y conocerlos a } \\
\text { todos en profundidad. Y posteriormente creo que sí, que puede ser } \\
\text { bastante útil. } \\
\text { La pantalla genera frialdad... somos más de contacto. }\end{array}$ \\
\hline
\end{tabular}

Red temática 3. Tema global: Lo que subyace a las prácticas de Atención Temprana de teleintervención

Este tema incluye cuestiones relativas al enfoque desde el que se entiende la intervención: las experiencias recogidas se pueden situar en un continuo que abarca desde un enfoque más orientado al niño y sus déficits hasta un enfoque centrado en la familia y prácticas recomendadas, junto con el rol profesional, la organización de los equipos, la implementación y la tendencia a reproducir a través de la teleintervención los enfoques previos al confinamiento.

Entre las categorías analizadas en este tema global encontramos las siguientes. Empoderamiento familiar: competencia y confianza, recursos y materiales. Colaboración familia-profesional: vínculo, cercanía-confianza-intimidad, construir verdadero equipo familia-profesional (relación de igualdad), disponibilidad. Formación ECF: agradecimiento a Plena inclusión y a AEPI por la formación continua y adaptada en tiempo de crisis, formación-reflexión, revisión en grupos/redes colaborativas. Percepción del desarrollo de los niños: percepción optimista, tiempo que paso con mis niños. Apoyos: rutinas/desarrollo, conducta, apoyo emocional y apoyo escolar. La TI ha intentado reproducir el enfoque que había previamente: la teleintervención promueve el enfoque centrado en familias, las prácticas centradas en la familia hacen más fácil la teleintervención (continuidad a las prácticas previas), otros enfoques encuentran más dificultades. 
Figura 4. Categorías en la red temática 3: Lo que subyace a la teleintervención

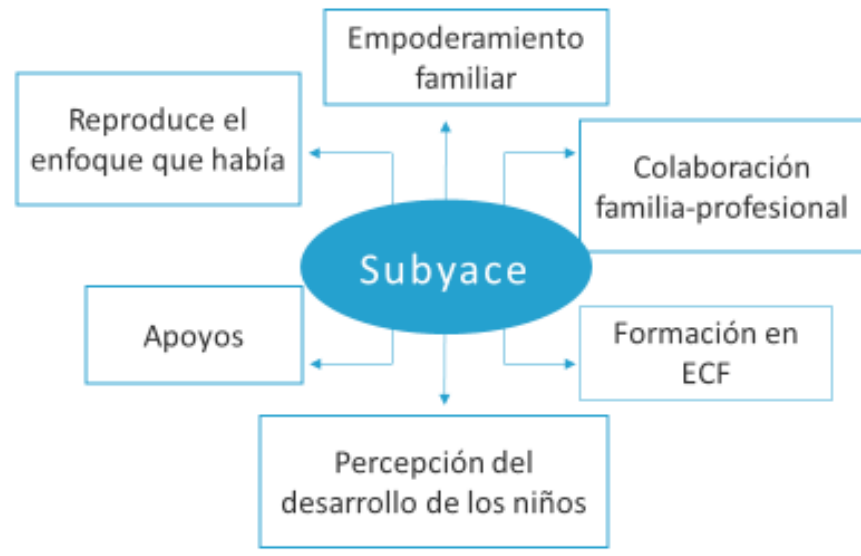

Por último, en la Tabla 6 se presentan los resultados de la última categoría analizada, lo que subyace a la teleintervención en las Prácticas de Atención Temprana.

\begin{tabular}{|c|l|}
\hline \multicolumn{2}{|c|}{$\begin{array}{c}\text { TABLA 6. Categorías analizadas y extractos } \\
\text { en la red temática 3: Lo que subyace a la teleintervención }\end{array}$} \\
\hline Categorías & \multicolumn{1}{c|}{ Extractos de las narrativas } \\
\hline $\begin{array}{l}\text { Percepción del } \\
\text { desarrollo de } \\
\text { los niños/as }\end{array}$ & $\begin{array}{l}\text { Ha habido muchos padres que se han dado cuenta de muchas cosas } \\
\text { que son capaces de hacer sus hijos y que no habían reparado en ello, } \\
\text { porque a lo mejor pasaban la mañana en la guarde o como que se } \\
\text { olvidaban un poco de esa parte. } \\
\text { Yo me he dado cuenta de que mi hija, como todos los niños, son } \\
\text { capaces de bastante más de lo que yo esperaba. }\end{array}$ \\
\hline Apoyos & $\begin{array}{l}\text { Antes me preocupaba más el lenguaje y ahora me preocupa más el } \\
\text { tema de comida [...] y en parte el de caminar, se cae menos, pero } \\
\text { tampoco camina mucho por la parcela. No la dejo que camine } \\
\text { mucho. } \\
\text { Lo que más hemos dado es tema conductual. Al estar encerrados } \\
\text { pues a muchos papis, pues bueno, los niños estaban alterados y los } \\
\text { papás también. Era una situación difícil, entonces ha salido mucho } \\
\text { tema de conducta. } \\
\text { El primer apoyo era más psicológico y emocional de apoyar a la } \\
\text { familia, que te cuente toda la situación que les desbordaba y demás. }\end{array}$ \\
\hline
\end{tabular}




\begin{tabular}{|c|c|}
\hline \multicolumn{2}{|r|}{$\begin{array}{l}\text { Tabla 6. Categorías analizadas y extractos } \\
\text { en la red temática 3: Lo que subyace a la teleintervención (cont.) }\end{array}$} \\
\hline Categorías & Extractos de las narrativas \\
\hline \multirow[t]{8}{*}{$\begin{array}{l}\text { La TI repro- } \\
\text { duce el mode- } \\
\text { lo que había } \\
\text { previam. }\end{array}$} & $\begin{array}{l}\text { Yo la verdad no estaba trabajando con modelo centrado en familia. } \\
\text { Nos ha venido bien, entre comillas, la pandemia, porque hemos em- } \\
\text { pezado la transformación en el centro. Incluíamos a las familias en } \\
\text { la sesión, trabajábamos con la familia dentro, con el niño y ahora ya } \\
\text { nos hemos reciclado. }\end{array}$ \\
\hline & $\begin{array}{l}\text { Nos hemos quitado una barrera que había, una barrera que no se veía } \\
\text { y ahora las familias dejan que trabajemos más íntimamente con ellos. }\end{array}$ \\
\hline & $\begin{array}{l}\text { Me planteaba también de qué manera la teleintervención o esta situa- } \\
\text { ción ha puesto en valor el peso del enfoque, ahora más que nunca se } \\
\text { ha visto el sentido que tenía. }\end{array}$ \\
\hline & $\begin{array}{l}\text { Con la edad que tiene, lo que le viene bien es interactuar con el per- } \\
\text { sonal, con la logopeda. Entonces, a través de una pantalla ya no pres- } \\
\text { ta la atención que debe prestar y está a su rollo. }\end{array}$ \\
\hline & $\begin{array}{l}\text { Cuando la logopeda está aquí Mónica la ve, la siente, está pendiente } \\
\text { de ella y como que se comporta de otra manera. Ahora mismo pues, } \\
\text { está tirada en el sofá o se va a un cuarto a jugar o lo que sea. Cuando } \\
\text { viene la logopeda a casa, ella sabe dónde está su silla, se sienta en su } \\
\text { silla y hace sus cosas ahí (luego ellos ponen en práctica lo que han } \\
\text { trabajado durante la sesión). }\end{array}$ \\
\hline & $\begin{array}{l}\text { Pues ha sido una ocasión de oro para que las familias se metan de lle- } \\
\text { no, las que no estaban convencidas, se metan de lleno en el programa } \\
\text { centrado en el trabajo en entorno natural. }\end{array}$ \\
\hline & $\begin{array}{l}\text { Nosotras trabajamos en prácticas centradas en la familia, con lo cual } \\
\text { trabajamos en el entorno, y, para mí, la intervención ha sido un me- } \\
\text { dio. Ha sido un medio para poder dar continuidad a la intervención } \\
\text { en esta situación extraña, con lo cual lo valoro de manera positiva } \\
\text { porque, frente a otros servicios, nuestras familias han podido seguir } \\
\text { con la intervención que estaban recibiendo. }\end{array}$ \\
\hline & $\begin{array}{l}\text { Nosotras que estamos en el cambio de transformación al modelo, ha } \\
\text { sido como un empujón, y como coger fuerzas realmente, y también } \\
\text { desde la propia práctica. Las familias están entendiendo muy bien el } \\
\text { proceso, cosa que se agradece bastante. }\end{array}$ \\
\hline
\end{tabular}




\begin{tabular}{|c|l|}
\hline \multicolumn{2}{|c|}{ TABLA 6. Categorías analizadas y extractos } \\
\hline en la red temática 3: Lo que subyace a la teleintervención (cont.)
\end{tabular}




\begin{tabular}{|l|l|}
\hline \multicolumn{2}{|c|}{ TABLA 6. Categorías analizadas y extractos } \\
\hline en la red temática 3: Lo que subyace a la teleintervención (cont.)
\end{tabular}




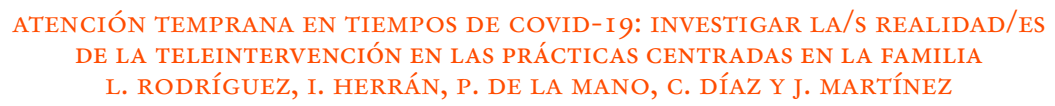

Una vez expuestos los resultados obtenidos tras el análisis de las tres redes temáticas, acompañados de las categorías que originan los temas globales y su descripción, se presenta la discusión de estos resultados.

\section{Discusión}

No aprendemos de la experiencia, sino de la reflexión sobre la experiencia. John Dewey

Este apartado intenta organizar los datos, por un lado, en relación con los objetivos del estudio y, por otro, en relación con el diálogo con otros trabajos, si bien son limitadas las publicaciones comparables con el contexto social en el que se desarrolla el trabajo.

La metodología utilizada en el estudio fue de corte fenomenológico, que tiene como objetivo identificar el significado real de una experiencia como se entiende por la persona que lo experimenta (Cresswell et al., 2007). Así, podemos relacionar las narrativas dando respuesta a los objetivos de investigación.

Objetivo 1. Explorar la percepción de las familias ante la atención recibida mediante la teleintervención. En relación con las cuestiones que se derivan de este trabajo destacan, en primer lugar, la resiliencia y la rápida capacidad de adaptación al cambio de profesionales y familias ante la situación de confinamiento. Las familias declaran sentirse muy apoyadas durante todo el proceso. Además, los profesionales manifiestan diferentes niveles de estrés propio y de las familias en función del grado de disonancia ante la nueva situación. Para las familias que ya realizaban PCF el estrés de adaptación a una nueva herramienta parece haber tenido un menor impacto, de acuerdo con sus narrativas.

Un análisis reciente (McCarthy et al., 2020) trabaja a partir de la percepción de las familias y revela que ciertos cuidadores o las características del niño sí influyeron en algunos aspectos de las creencias de los cuidadores sobre su autoeficacia o su participación en la teleintervención. Estas cuestiones también se evidencian en nuestro estudio.

Objetivo 2. Explorar la percepción de los profesionales ante la atención of recida mediante la teleintervención. Parece existir una variabilidad de apoyo de las entidades a sus profesionales (en cuestiones como, por ejemplo, la cesión de los recursos tecnológicos propios para uso profesional), aunque hay bastante coincidencia en que la intervención requiere recursos adecuados que deben ser aportados por el centro. Los profesionales destacan como factor protector la importancia del equipo de cara a resolver las dificultades que surgen ante una situación de crisis global. "No hubiera sido posible sin el equipo" es una frase presente en las narrativas de los profesionales en todos los grupos focales. No deja de ser emocionante que los equipos, que tanto se implican en el apoyo y el cuidado de las familias, también se sientan cuidados entre 
ATENCIÓN TEMPRANA EN TIEMPOS DE COVID-I 9: INVESTIGAR LA/S REALIDAD/ES DE LA TELEINTERVENCIÓN EN LAS PRÁCTICAS CENTRADAS EN LA FAMILIA

L. RODRÍGUEZ, I. HERRÁN, P. DE LA MANO, C. DÍAZ Y J. MARTÍNEZ

ellos. Es importante comprender a un nivel interno que el modelo de liderazgo que sostiene el cuidado del equipo es una variable clave en las prácticas recomendadas y en las prácticas de Atención Temprana.

En otro estudio similar (McCarthy et al., 2020), esta vez centrado en la autoevaluación de los profesionales sobre las prácticas centradas en la familia en teleintervención y en atención presencial, los resultados indicaron que no había diferencias significativas entre la teleintervención y las sesiones de atención presencial con respecto a la autoevaluación de los profesionales sobre su uso de prácticas centradas en la familia.

Objetivo 3. Explorar la percepción de los coordinadores de centros ante la atención ofrecida mediante la teleintervención. La reflexión en la práctica, la formación recibida y el análisis global de la situación han resultado ser factores clave para la supervivencia (sic) de profesionales y coordinadoras en este periodo. La trayectoria en PCF parece amortiguar todo el temblor que han supuesto el confinamiento y la teleintervención. Las PCF han permitido estar también ofreciendo los apoyos demandados durante la teleintervención en la medida en que la actuación profesional ha tenido a la familia como referente principal. En los casos en los que se han intentado mantener intervenciones directas sobre el niño, tanto familias como profesionales refieren más dificultades. En esta línea, un estudio reciente (Klatte et al., 2020) identifica cuatro temas en la descripción de las experiencias de los profesionales con el compromiso de los padres: comprensión mutua, creación de una relación constructiva entre el profesional y el padre o madre, el empoderamiento de las familias y las barreras que encontraban. Las necesidades de formación en PCF (que en el estudio se valoran muy positivamente), tanto en prácticas relacionales y prácticas participativas como en reflexión-acción, pensamiento crítico y estrategias de acompañamiento familiar, formando a los profesionales en la mejor manera de implicar a los padres, centrándose en la comprensión mutua, creando relaciones constructivas entre el profesional y las familias y empoderando a estas, resultan claves para la teleintervención en PCF (Klatte et al., 2020). Parece haber un consenso en los estudios al poner de relieve la falta de cualificación de los profesionales para llevar a cabo programas de Atención Temprana mediante la teleintervención para apoyar verdaderamente a las familias (Houston y Stredler-Brown, 2012; Behl y Houston, 2012; Grogan-Johnson et al., 2015), y para evaluar la percepción y grado de satisfacción de familias y profesionales utilizando teleintervención.

Objetivo 4. Conocer en profundidad barreras y facilitadores de este medio de apoyo en Atención Temprana. Ninguna de las narrativas analizadas pone en duda el impacto que tienen las familias sobre sus hijos e hijas. Incluso profesionales con una menor trayectoria en transformación hacia PCF comparten que la teleintervención ha resultado provechosa para hacer el cambio de enfoque; aprovechando la teleintervención han revisado y actualizado sus prácticas. Además, tanto profesionales como familias declaran que, una vez conocido este enfoque en el que las necesidades de la familia son el motor de arranque y el eje absoluto, no volverían a un modelo tradicional. Recordamos al hilo de esto unas palabras del Dr. Dunst (en comunicación 
ATENCIÓN TEMPRANA EN TIEMPOS DE COVID-I 9: INVESTIGAR LA/S REALIDAD/ES DE LA TELEINTERVENCIÓN EN LAS PRÁCTICAS CENTRADAS EN LA FAMILIA

L. RODRÍGUEZ, I. HERRÁN, P. DE LA MANO, C. DÍAZ Y J. MARTínez

privada) en las que afirma que cualquier práctica es susceptible de llegar a ser PCF. Dunst subraya, de hecho, el valor de los profesionales que aún están a medio camino, pero que, innegablemente, ya han comenzado.

Otros estudios que utilizan una aproximación cualitativa, como Cason (2009) o el del equipo de Little et al. (2015), a través del diseño de grupos focales con familias y entrevistas a informantes clave, se acercan a la comprensión de las barreras a los servicios de Atención Temprana para los bebés prematuros, a través de la teoría fundamentada. El estudio de Cason (2011) utiliza, además, el análisis de redes temáticas en las que identifica tres categorías relacionadas con los beneficios/fortalezas, desafíos/debilidades y recomendaciones para mejorar el programa de Atención Temprana. Los resultados de la evaluación del programa indicaron que la teleintervención tiene el potencial de satisfacer de manera rentable las necesidades terapéuticas de los niños que viven en zonas rurales donde hay escasez de centros o servicios. En esta línea, el trabajo de Ekberg et al. (2019) señala que el aislamiento geográfico puede agravarse aún más cuando los niños no tienen acceso a oportunidades de aprendizaje que puedan facilitar el desarrollo de la competencia comunicativa y el diagnóstico de cualquier dificultad.

Objetivo 5. Valorar la incorporación de la teleintervención como un medio más en las prácticas de Atención Temprana tras la crisis de la COVID-19. La teleintervención ha venido para quedarse, aunque con salvedades. Este parece ser otro punto clave y reflejado de manera explícita en las narrativas de la amplia mayoría de familias y profesionales, ya que, según relatan, la teleintervención amplía nuevas oportunidades de encuentro, funcionalidad, anticipación, salva distancias y ofrece resultados funcionales, si bien tiene su parte adversativa (sí, pero): requiere recursos; enfatiza la brecha tecnológica, lingüística y/o cultural, y tiene importantes limitaciones.

Un estudio de revisión sistemática, relacionado con la teleintervención en los logopedas (Molini-Avejonas et al., 2015), sostiene que la mayoría de los estudios revisados llegan a la conclusión de que el procedimiento de teleintervención tenía ventajas sobre el enfoque alternativo de no teleintervención. Lo relevante en este sentido es que no hay una respuesta única, ya que la teleintervención parece no haber impactado de una sola manera en las familias o en los profesionales. El trabajo de Houston y Stredler-Brown (2012) afirma que la opción de la teleintervención garantiza que todas las familias reciben la intervención a la que tienen derecho si no hay otra alternativa. En todo caso en el presente trabajo se evidencia una opinión general de que la teleintervención sirve como complemento, pero no sustituye a la presencialidad.

\section{Conclusiones}

El trabajo describe un proceso de exploración de las prácticas centradas en la familia en los primeros meses del confinamiento, prácticas que se han dirigido, en muchos casos, hacia la teleintervención. Los datos han sido recogidos mediante entrevistas etnográficas (a las familias) y grupos focales (con profesionales) y han sido tratados 
mediante análisis de redes temáticas. En este artículo se presentan los resultados del primer análisis temático realizado de acuerdo con los objetivos del estudio. Se pretende así ofrecer una mirada global -e intencionalmente descriptiva- de los datos, si bien en otros trabajos posteriores se espera profundizar en cada uno de los temas globales como entidad propia, así como en las consecuencias que subyacen para futuros diseños de prácticas centradas en la familia mediante la herramienta de teleintervención.

Los resultados muestran la percepción de las familias y los profesionales articulados en tres focos de redes temáticas: la reacción personal y colectiva al cambio, lo que puede permanecer de la teleintervención (con sus matices) y lo que subyace a las prácticas centradas en la familia en esta modalidad de teleintervención.

\subsection{Limitaciones del estudio}

El estudio se ha centrado en conocer la valoración de familias y profesionales sobre la teleintervención durante el confinamiento. Por esto, una limitación del mismo es no haber tenido en cuenta la opinión de aquellas familias que no han accedido por diversas razones a la teleintervención durante el confinamiento, bien por imposibilidad tecnológica o por decisión personal.

Las familias y profesionales que participan en el estudio pertenecen a centros y servicios de Atención Temprana de Castilla-La Mancha en proceso de transformación. Los participantes relatan sus experiencias durante el periodo de confinamiento y reflejan, mediante sus narrativas, la tendencia general que hemos recogido en este estudio. No obstante, la realidad señala que ha habido otras circunstancias y contingencias (como que no hayan participado familias que no han mantenido contacto durante el confinamiento con los centros y servicios de Atención Temprana) que pueden no haberse reflejado en este trabajo.

\subsection{Lineas futuras}

Este estudio abre, además, nuevas líneas de investigación futuras, como continuar analizando qué subyace de las afirmaciones referidas a la complejidad de transformación hacia unas verdaderas prácticas centradas en la familia. Así, en futuros trabajos se presentarían cuestiones con un nivel de interpretación más profundo, latente y/o dirigido a comprender procesos subyacentes en las prácticas en Atención Temprana que pudieran culminar en políticas específicas de apoyo a las PCF.

Emprender un viaje de transformación desde los servicios que tenemos hacia los servicios que se necesitan y que, éticamente, estamos comprometidos a ofrecer requiere de reflexión, conocimiento, compromiso y acción (Tamarit, 2015). Posibilitar y/o garantizar un acceso a las PCF en una posible situación de confinamiento futuro requiere, necesariamente, reflexión, conocimiento, compromiso y acción, así como una mirada hacia los protagonistas -tanto familias como profesionales- de la situación de crisis anterior. 


\section{ATENCIÓN TEMPRANA EN TIEMPOS DE COVID-I9: INVESTIGAR LA/S REALIDAD/ES DE LA TELEINTERVENCIÓN EN LAS PRÁCTICAS CENTRADAS EN LA FAMILIA L. RODRÍGUEZ, I. HERRÁN, P. DE LA MANO, C. DÍAZ Y J. MARTÍNEZ}

Este estudio, mediado por el contexto social en el que tuvo lugar en el que el confinamiento lo contaminó todo, ha pretendido dar voz a las narrativas de los protagonistas en esta difícil situación sociosanitaria global que vivimos desde marzo de 2020. La intención última de este trabajo es acercarse a la realidad o, mejor, las realidades que han vivenciado familias y profesionales desarrollando PCF mediante la teleintervención. Sirvan estos resultados para reflexionar sobre las prácticas y para facilitar las condiciones que permitan un impacto colectivo y transformador en la calidad de vida de las familias en el presente y en el futuro.

La pandemia de la COVID-19 nos ha vinculado con el origen y con la reacción de las sociedades para hacerle frente. La vivencia personal y social de dicha experiencia y de las fraternidades anudadas durante la crisis nos puede dar pie para encontrar un nuevo equilibrio en todos los planos []. ¿Habremos aprendido? ¿Seremos capaces de ponerlo en práctica? ¿Estamos todavía a tiempo? Esperemos que lo esencial que un virus ha puesto de manifiesto se mantenga visible ante nuestros ojos. (Fernando Conde, 2020: 418)

\section{Agradecimientos}

Manifestamos nuestro profundo agradecimiento a las familias y profesionales que han participado generosamente en este estudio. Gracias a su experiencia, su mirada y sus narrativas ha sido posible acercarnos a explorar nuevas perspectivas que puedan contribuir a la calidad de vida de personas y colectivos, incluso en situación de crisis sociosanitaria.

\section{Declaración de conflicto de intereses}

Este estudio no ha recibido financiación externa. Los autores y autoras declaran no tener conflicto de intereses.

\section{Referencias bibliográficas}

Aharony, L. y Strasser, S. (1993). Parent satisfaction: what we know and what we still need to explore. Medical Care Review, 50(1), 226-248.

American association on intellectual and DEVElopmental Disabilities. (2010). Discapacidad Intelectual (11. ${ }^{\mathrm{a}}$ ed.). Alianza Editorial.

American speech-language-Hearing Association. (2008). Roles and responsibilities of speech-language pathologists in early intervention: position statement. Recuperado de: https://www.asha.org/policy/PS2008-00291/ https://doi:10.1044/policy.PS2008-00291 ASHA link: https://leader.pubs.asha.org/do/10.1044/how-our-early-intervention-practice-serves-families-through-telepractice/full/

Amor, A. M. y Verdugo, M. Á. (2018). Quality of life and its role for guiding practices in the social and educational services from the systems perspective. Tradycja I Wspótczesność 


\section{ATENCIÓN TEMPRANA EN TIEMPOS DE COVID-I 9: INVESTIGAR LA/S REALIDAD/ES DE LA TELEINTERVENCIÓN EN LAS PRÁCTICAS CENTRADAS EN LA FAMILIA \\ L. RODRÍGUEZ, I. HERRÁN, P. DE LA MANO, C. DÍAZ Y J. MARTínEZ}

Pedagogiki Specjalnej W Tworzeniu Społeczeństwa Dla Wszystkich, 3(41), 15-17. https:// doi:10.5604/01.3001.0012.7817

Ashworth, M. y BAKer, A. (2000). "Time and space": carer's views about respite care. Health and Social Care in the Community, 8(1), 50-56. https://doi.org/10.1046/j.13652524.2000.00221.x

Attride-Stirling, J. (2001). Thematic networks: an analytic tool for qualitative research. Qualitative Research, 1(3), 385-405. https://doi.org/10.1177/146879410100100307

BAHARAv, E. y ReISER, C. (2010). Using telepractice in parent training in early autism. Telemedicine and e-Health, 16(6), 727-731. https://doi.org/10.1089/tmj.2010.0029

Band, S., Lindsay, G., Law, J., Soloff, N., Peacey, N., Gascoigne, M. y Radford, J. (2002). Are health and education talking to each other? Perceptions of parents of children with speech and language needs. European Journal of Special Needs Education, 17(3), 211-227. https://doi.org/10.1080/08856250210162121

Barnett, W. S. y Hustedt, J. J. (2005). Head start's lasting benefits. Infants and Young Children, 18, 16-24.

Baxendale, J., Frankham, J. y Hesketh, A. (2001). The Hanen Parent Programme: a parent's perspective. International Journal of Language and Communication Disorders, 36(Supplement 2001), 511-516. https://doi.org/10.3109/13682820109177938

Behl, D. D., Blaiser, K., Cook, G., Barrett, T., Callow-Heusser, C., Brooks, B. M., Dawson, P., Quigley, S. y White, K. R. (2017). A multisite study evaluating the benefits of early intervention via telepractice. Infants and Young Children, 30(2), 147-161. https:// doi.org/10.1097/IYC. 0000000000000090

Behl, D. D., Houston, T. y Stredler-Brown, A. (2012). The value of a learning community to support telepractice for infants and toddlers with hearing loss. Volta Review, 112(3), 313-327. https://doi:10.17955/tvr.112.3.m.701

Blaiser, K. M., Behl, D., Callow-Heusser, C. y White, K. R. (2013). Measuring costs and outcomes of tele-intervention when serving families of children who are deaf/hardof-hearing. International Journal of Telerehabilitation, 5(2), 3. https://doi.org/10.5195/ ijt.2013.6129

Blake H. L (2020). Intelligibility enhancement via telepractice during COVID-19 restrictions. Perspectives of the ASHA Special Interest Groups, 5(6), 1797-1800. https://doi. org/10.1044/2020_PERSP-20-00133

Boisvert, M., Lang, R., Andrianopoulos, M. y Boscardin, M. L. (2010). Telepractice in the assessment and treatment of individuals with autism spectrum disorders: a systematic review. Developmental Neurorehabilitation, 13(6), 423-432. https://doi.org/10.3109/1751 8423.2010.499889

Braun, V. y Clarke, V. (2006). Using thematic analysis in psychology. Qualitative Research in Psychology, 3(2), 77-101. https://doi.org/10.1191/1478088706qp063oa

BRETT, J. (2002). The experience of disability from the perspective of parents of children with profound impairment: is it time for an alternative model of disability? Disability and Society, 17(7), 825-843. https://doi.org/10.1080/0968759022000039109

Brown, J. (2011). ASHA and the evolution of telepractice. Perspectives on Telepractice, 1(1), 4-9. https://doi.org/10.1044/tele1.1.4

Bruder, M. B. (2000). Family-centered early intervention: clarifying our values for the new millennium. Topics in Early Childhood Special Education, 20(2), 105-115. https://doi. org/10.1177/027112140002000206 


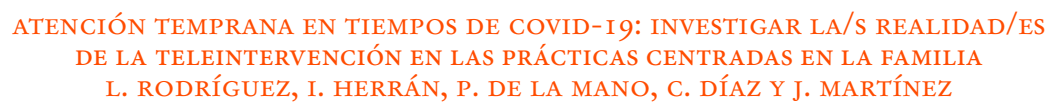

Carpenedo, D. J. (2006). Telepractice in the city: the story of the visiting nurse service of New York home care. The ASHA Leader, 11(14), 10-11. https://doi.org/10.1044/leader. FTR4.11142006.10

Cason, J. (2009). A pilot telerehabilitation program: delivering early intervention services to rural families. International Journal of Telerehabilitation, 1(1), 29. https://doi.org/10.5195/ ijt.2009.6007

Conde Gutiérrez Del Álamo, F. (2020). Lo esencial es invisible a los ojos: crónica de la pandemia de la COVID-19. CIMOP.

Constantinescu, G., Waite, M., Dornan, D., Rushbrooke, E., Brown, J., McGovern, J., Ryan, M. y Hill, A. (2014). A pilot study of telepractice delivery for teaching listening and spoken language to children with hearing loss. Journal of Telemedicine and Telecare, 20(3), 135-140. https://doi.org/10.1177/1357633X14528443

Constitución Española. (1978). Boletín Oficial del Estado, 29 de diciembre de 1978, Madrid, España, Cortes Generales, 29, 311, 29313-29424.

Courtney, E., Kiernan, G., Guerin, S., Ryan, K. y Mcquillan, R. (2018). Mothers' perspectives of the experience and impact of caring for their child with a life-limiting neurodevelopmental disability. Child: Care, Health, and Development, 44(5), 704-710. https://doi. org/10.1111/cch.12580

Creswell, J. (2007). Qualitative inquiry and research design - choosing among five approaches (2. ${ }^{\mathrm{a}}$ ed.). Sage Publications.

Creswell, J. W., Hanson, W. E., Clark Plano, V. L. y Morales, A. (2007). Qualitative research designs: selection and implementation. The Counselling Psychologist, 35(2), 236-264. https://doi.org/10.1177/0011000006287390

Curtiss, S. L., Mcbride, B. A., Uchima, K., Laxman, D. J., Santos, R. M., WeglarzWARD, J. y KERN, J. (2019). Understanding provider attitudes regarding father involvement in Early Intervention. Topics in Early Childhood Special Education. https://doi. org/10.1177/0271121419844829

Dalmau, M., Balcells, A., Giné, C. G., Cañadas, M., Casas, O., Cuscó, Salat, Y., FaRRÉ, V. y CAlaf, N. (2017). Cómo implementar el modelo centrado en la familia en Atención Temprana. Anales de Psicología/Annals of Psychology, 33(3), 641-651. https://doi. org/10.6018/analesps.33.3.263611

Decreto 88/2017, de 5 de diciembre, por el que se regulan las condiciones mínimas exigibles a los centros y servicios destinados a la atención a personas con discapacidad en Castilla-La Mancha (2007). Diario Oficial de Castilla-La Mancha, 247, sección I, de 26 de diciembre de 2017, 32651-32695. https://docm.castillalamancha.es/portaldocm/descargarArchivo. do?ruta=2017/12/26/pdf/2017_14922.pdf\&tipo=rutaDocm

Delaunay, R. (1912). Light The Structurist, 13, 43.

Division for EARly ChIldhood (DEC). (2014). Recommended practices in early intervention/early childhood special education 2014 [Monograph]. Recuperado de http://www.decsped.org/recommendedpractices

Division For EARly ChILdhood. (2018). DEC Recommended practices: a quick overview. Recuperado de http://www.dec-sped.org/dec-recommended-practices

Duchan, J. (2001). Impairment and social views of speech pathology: clinical practices revisited. Advances in Speech-Language Pathology, 3(1), 37-45. https://doi. org/10.3109/14417040109003707

Duchan, J. (2004). Framework in language and literacy: how theory informs practice. The Guilford Press. 
ATENCIÓN TEMPRANA EN TIEMPOS DE COVID-I9: INVESTIGAR LA/S REALIDAD/ES DE LA TELEINTERVENCIÓN EN LAS PRÁCTICAS CENTRADAS EN LA FAMILIA

L. RODRÍGUEZ, I. HERRÁN, P. DE LA MANO, C. DÍAZ Y J. MARTíneZ

Dulguérian, M. R. (2012). Experiencia emocional de los padres de niños nacidos prematuros y aspectos psicológicos. EMC-Pediatría, 47(3), 1-6. https://doi.org/10.1016/S12451789(12)62751-6

Dunst, C. J., Boyd, K., Trivette, C. y Hamby, D. (2002). Family-oriented program models and professional Helpgiving Practices. Family Relations, 51(3), 221-229.

Dunst, C. J., Bruder, M. B. y Espe-Sherwindt, M. (2014). Family capacity-building in Early Childhood Intervention: do context and setting matter? School Community Journal, 24(1), 37-48.

Dunst, C. J., Bruder, M. B., Trivette, C. M., Hamby, D., Raab, M. y Mclean, M. (2001). Characteristics and consequences of everyday natural learning opportunities. Topics in Early Childhood Special Education, 21, 68-92. https://doi.org/10.1177\%2F027112140102100202

Dunst, C. J. y TrivetTe, C. M. (1996). Empowerment, effective help-giving practices and family-centered care. Pediatric Nursing, 22(4), 334-337, 343.

Dunst, C. J., Espe-Sherwindt, M. y Намby, D. W. (2019). Does capacity-building professional development engender practitioners' use of capacity-building family-centered practices? European Journal of Educational Research, 8(2), 515-526. https://doi.org/10.12973/ eu-jer.8.2.513

Dunst, C. J. y TrivetTe, C. M. (2009). Capacity-building family systems intervention practices. Journal of Family Social Work, 12 (2), 119-143. https://doi.org/10.1080/10522150802713322

Dunst, C. J. y Trivette, C. M. y Hamby, D. W. (2007). Metanalysis of family-centered helpgiving practices research. Mental Retardation and Developmental Disabilities Research Reviews, 13, 370-378. https://doi.org/10.1002/mrdd.20176

Edmunds, C. y Staniszewska, S. (2000). Accessing the user's perspective. Health and Social Care in the Community, 8(6), 417-424. https://doi.org/10.1046/j.1365-2524.2000.00267.x

Edmunds, H. (1999). The focus group research handbook. The Bottom Line.

Edwards, M., Stredler-Brown, A. y Houston, K. T. (2012). Expanding use of telepractice in speech-language pathology and audiology. Volta Review, 112(3), 227-242. https:// doi:10.17955/tvr.112.3.m.704

Ekberg, S., Houen, S., Fisher, B., Theobald, M., Danby, S., Ekberg, S. y Grove, K. (2019). Engaging young children in speech and language therapy via videoconferencing. Everyday Technologies in Healthcare, 175-192.

Escartí, A., Boronat, N., Llopis, R., Torres, R. y Vento, M. (2016). Estudio piloto sobre el estrés y la resiliencia familiar en recién nacidos prematuros. Anales de Pediatría, 84(1), 3-9. https://doi.org/10.1016/j.anpedi.2015.03.001

Escobar, J. y Bonilla, F. (2017). Grupos focales: una guía conceptual y metodológica. Cuadernos Hispanoamericanos de Psicología, 9(1), 51-67.

Escorcia-Mora, C. T., García-Sánchez, F. A., Sánchez-López, M. C., Orcajada-Sánchez, N. y Hernández-Pérez, E. (2018). Prácticas de Atención Temprana en el sureste de España: perspectiva de profesionales y familias. Anales de PsicologialAnnals of Psychology, 34(3), 500-509. https://doi.org/10.6018/analesps.34.3.311221

EsPe-SHERwindt, M. (2008). Family-centred practice: collaboration, competency and evidence. Support for Learning, 23(3), 136-143. https://doi:10.1111/j.1467-9604.2008.00384.x

Finch, H. y Lewis, J. (2006). Focus groups. En J. Ritchie y J. Lewis (Eds.), Qualitative research practice - a guide for social science students and researchers. Sage Publications.

García-Grau, P., Martínez-Rico, G., McWilliam, R. A. y Cañadas Pérez, M. (2020). Typical and ideal practices in early intervention in Spain during a transformation pro-

Ediciones Universidad de Salamanca / CC BY-NC-ND

Siglo Cero, vol. 52, número extraordinario, 2021, pp. 75-117 
ATENCIÓN TEMPRANA EN TIEMPOS DE COVID-I 9: INVESTIGAR LA/S REALIDAD/ES DE LA TELEINTERVENCIÓN EN LAS PRÁCTICAS CENTRADAS EN LA FAMILIA

L. RODRÍGUEZ, I. HERRÁN, P. DE LA MANO, C. DÍAZ Y J. MARTíneZ

cess of professional practices. Journal of Early Intervention, 42(1), 3-19. https://doi. org $/ 10.1177 \% 2 F 1053815119859046$

García Sánchez, F. A. (2020). Atención Temprana, prácticas centradas en la familia y logopedia. Revista de Logopedia, Foniatría y Audiología, 40(3), 95-98. https://doi.org/10.1016/j. rlfa.2020.06.001

García-Sánchez, F. A., Escorcia-Mora, C. T. y Franco Castellano, I. (2020). Reflexiones y estrategias de acompañamiento familiar en Atención Temprana. Revista de Logopedia, Foniatría y Audiología, 40(3), 118-127. https://doi.org/10.1016/j.rlfa.2020.02.004

García-Sánchez, F. A., Escorcia, C. T., Sánchez-López, M. C., Orcajada, N. y HernánDez-PÉrez, E. (2014). Atención Temprana centrada en la familia. Siglo Cero, 45(3), 6-27.

García Sánchez, F. A., Gómez, N. R., Sánchez, N. O., Mora, C. T. E. y Pérez, M. C. (2018). Necesidades de formación en prácticas centradas en la familia en profesionales de Atención Temprana españoles. Bordón. Revista de Pedagogía, 70(2), 39-55. https://doi. org/10.13042/Bordon.2018.59913

Gat (2000). Libro Blanco de la Atención Temprana. Real Patronato sobre Discapacidad.

Gat (2011). La Realidad de la Atención Temprana en España. Real Patronato sobre Discapacidad.

Glogowska, M. (2002). Time to talk-parent's accounts of children's speech difficulties. Whurr Publisher.

Glogowska, M. y Campbell, R. (2000). Investigating parental views of involvement in preschool speech and language therapy. International Journal of Language and Communication Disorders, 35(3), 391-405. https://doi.org/10.1080/136828200410645

Gray, P. H., Edwards, D. M., O’Callaghan, M. J. y Cuskelly, M. (2012). Parenting stress in mothers of preterm infants during early infancy. Early Human Development, 88(1), 4549. https://doi.org/10.1016/j.earlhumdev.2011.06.014

Grogan-Johnson, S., Meehan, R., McCormick, K. y Miller, N. (2015). Results of a national survey of preservice telepractice training in graduate speech-language pathology and audiology programs. Contemporary Issues in Communication Science and Disorders, 42, 122-137. https://doi.org/10.1044/cicsd_42_S_122

Grogan-Johnson, S., Schmidt, A. M., Schenker, J., Alvares, R., Rowan, L. E. y Taylor, J. (2013). A comparison of speech sound intervention delivered by telepractice and side-byside service delivery models. Communication Disorders Quarterly, 34(4), 210-220. https:// doi.org/10.1177\%2F1525740113484965

Guba, E. G. y Lincoln, Y. S. (1994). Competing paradigms in qualitative research. En N. K. Denzin y Y. S. Lincoln (Eds.), Handbook of qualitative research (pp. 105-117). Sage.

Guralnick, M. J. (2001). A developmental systems model for early intervention. Infants and Young Children, 14(2), 1-18.

Guthrie, S. y Stansfield, J. (2015). Teatime threats. Choking incidents at the evening meal. Journal of Applied Research in Intellectual Disabilities, 30(1), 47-60. https://doi. org/10.1111/jar.12218

Han, J. Y. (2011). Transaction logfile analysis in health communication research: challenges and opportunities. Patient Education and Counselling, 82(3), 307-312. https://doi. org/10.1016/j.pec.2010.12.018

Hedge, M. N. y Maul, C. A. (2006). Language disorders in children - an evidence-based approach to assessment and treatment. Pearson Education Inc.

Heimdahl, E. C. y Serrano, A. M. (2020). Video-supported assessment as a method of bridging families and caregivers with professionals in Early Intervention. Revista de Logopedia, Foniatría y Audiología, 40(3), 101-109. https://doi.org/10.1016/j.rlfa.2020.01.002

Ediciones Universidad de Salamanca / CC BY-NC-ND

Siglo Cero, vol. 52, número extraordinario, 2021, pp. 75-117 


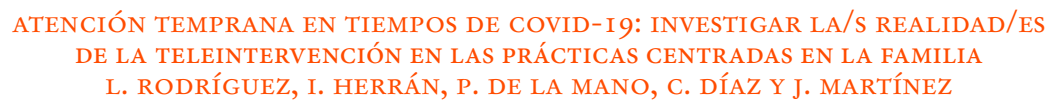

Houston, K. T. y Stredler-Brown, A. (2012). A Model of Early Intervention for children with hearing loss provided through telepractice. Volta Review, 112(3). https://doi:10.17955/ tvr.112.3.m.710

Houston, K. T., Stredler-Brown, A. y Alverson, D. C. (2012). More than 150 years in the making: the evolution of telepractice for hearing, speech, and language services. Volta Review, 112(3). https://doi/pdf/10.1044/persp1.SIG9.60

Hughes-Scholes, C. H. y Gavidia-Payne, S. (2016). Development of a routines-based early childhood intervention model. Educar em Revista, 59, 141-154. https://doi. org/10.1590/0104-4060.44616

Jaramillo, M. F., Mora, C. T. E., Ortiz, E., Chalén, D. F. Q., Legarda, P. O. y Boyce, L. (2020). Intervención basada en rutinas como apoyo a la participación familiar para el desarrollo del lenguaje en bebés prematuros. Revista de Logopedia, Foniatría y Audiología, 40(3), 110-117. https://doi.org/10.1016/j.rlfa.2020.02.005

Juan, S. y Roussos, A. (2010). El focus group como técnica de investigación cualitativa. Documento de Trabajo n. ${ }^{\circ} 256$. Universidad de Belgrano. Disponible en: http://repositorio. ub.edu.ar/bitstream/handle/123456789/4781/254_Roussos.pdf?sequen

Karmiloff-Smith, A. y Thomas, M. (2003). What can developmental disorders tell us about the neurocomputational constraints that shape development? The case of Williams syndrome. Development and Psychopathology, 15(4), 969-990. https://doi.org/10.1017/ S0954579403000476

Kellar-Guenther, Y., Rosenberg, S. A., Block, S. R. y Robinson, C. C. (2014). Parent involvement in early intervention: what role does setting play? Early Years, 34(1), 81-93. https://doi.org/10.1080/09575146.2013.823382

Kelso, G. L., Fiechtl, B. J., Olsen, S. T. y Rule, S. (2009). The feasibility of virtual home visits to provide early intervention: a pilot study. Infants and Young Children, 22(4), 332340. https://doi:10.1097/IYC.0b013e3181b9873c

Kiernan, J. (2013). Family and professional perspectives on the lived experience of children with a learning disability and behavioural needs. Doctoral dissertation. Manchester Metropolitan University.

Klatte, I. S., Harding, S. y Roulstone, S. (2019). Speech and language therapists' views on parents' engagement in parent-child interaction therapy (PCIT). International Journal of Language and Communication Disorders, 54(4), 553-564. https://doi.org/10.1111/14606984.12459

Klatte, I. S., Lyons, R., Davies, K., Harding, S., Marshall, J., McKean, C. y Roulstone, S. (2020). Collaboration between parents and SLTs produces optimal outcomes for children attending speech and language therapy: gathering the evidence. International Journal of Language and Communication Disorders, 1. https://doi.org/10.1111/1460-6984.12538

Kohnert, K. (2008). Language disorders in bilingual children and adults. Plural Publishing.

Kovarsky, D., Duchan, J. y Maxwell, M. (Eds.). (1999). Co-constructing (in) competence: disabling evaluations in clinical and social interaction. Erlbaum.

Krueger, E. y Reilly, D. (2011). The cash or care conflict in general practice: a cautionary tale with methodological reflections. Patient Education and Counseling, 82(3), 479-481. https://doi.org/10.1016/j.pec.2011.01.019

Levickis, P., McKean, C., Walls, E. y Law, J. (2020). Training community health nurses to measure parent-child interaction: a mixed-methods study. European Journal of Public Health, 30(3), 426-431. https://doi.org/10.1093/eurpub/ckz155 


\section{ATENCIÓN TEMPRANA EN TIEMPOS DE COVID-I9: INVESTIGAR LA/S REALIDAD/ES DE LA TELEINTERVENCIÓN EN LAS PRÁCTICAS CENTRADAS EN LA FAMILIA \\ L. RODRÍGUEZ, I. HERRÁN, P. DE LA MANO, C. DÍAZ Y J. MARTÍNEZ}

Levickis, P., McKean, C., Wiles, A. y Law, J. (2020). Expectations and experiences of parents taking part in parent-child interaction programmes to promote child language: a qualitative interview study. International Journal of Language and Communication Disorders. https://doi.org/10.1111/1460-6984.12543

Litosseliti, L. (2003). Using focus groups in research. New York.

Little, A. A., Kamholz, K., Corwin, B. K., Barrero-Castillero, A. y Wang, C. J. (2015). Understanding barriers to early intervention services for preterm infants: lessons from two states. Academic Pediatrics, 15(4), 430-438. https://doi.org/10.1016/j.acap.2014.12.006

Lyons, R. y Kinneen, L. (2007/2008). A case for contracting in speech and language therapy. Journal of Clinical Speech and Language Studies, 16(94-101).

Lyons, R., O’Malley, M. P., O’Connor, P. y Monaghan, U. (2010). 'It's just so lovely to hear him talking': exploring the early-intervention expectations and experiences of parents. Child Language Teaching and Therapy, 26(1), 61-76. https://doi. org $/ 10.1177 \% 2$ F0265659009349975

Mahoney, G., Wheeden, C. A. y Perales, F. (2004). Relationship of preschool special education outcomes to instructional practices and parent-child interaction. Research in Developmental Disabilities, 25(6), 539-558. https://doi.org/10.1016/j.ridd.2004.04.001

Mansilla, R., Acosta, K. y Villazon, A. (2014). Prematurez: nociones relevantes y riesgo neurológico del prematuro. Revista Desafíos, 1(8), 51-60. Recuperado de http://revistas. ut.edu.co/index.php/desafios/article/view/461/387

Marco-Arenas, M., García-Sánchez, F. A. y Sánchez-López, M. C. (2020). Inteligencia emocional y prácticas participativas con la familia en Atención Temprana. RELIEVERevista Electrónica de Investigación y Evaluación Educativa, 26(1), 1-16. https://doi. org/10.7203/relieve.26.1.13168

Marshall, J. y Goldbart, J. (2008). 'Communication is everything I think'. Parenting a child who needs Augmentative and Alternative Communication (AAC). International Journal of Language and Communication Disorders, 43(1), 77-98. https://doi. org/10.1080/13682820701267444

Marshall, J., Goldbart, J. y Phillips, J. (2007). Parents' and speech and language therapists' explanatory models of language development. International Journal of Language and Communication Disorders, 42(5), 533-555. https://doi.org/10.1080/13682820601053753

Mas, J. M., Dunst, C. J., Hamby, D. W., Balcells-Balcells, A., García-Ventura, S., BAQUÉs, N. y GiNé, C. (2020). Relationships between family-centred practices and parent involvement in early childhood intervention. European Journal of Special Needs Education, 1-13. https://doi.org/10.1080/08856257.2020.1823165

Maxwell, J. A. (2008). Designing a qualitative study. The SAGE handbook of applied social research methods, 2, 214-253.

McCarthy, M., Duncan, J. y Leigh, G. (2012). Telepractice: the Australian experience in an international context. Volta Review, 112(3), 297-312.

McCarthy, M., Leigh, G. y Arthur-Kelly, M. (2019). Telepractice delivery of family-centred early intervention for children who are deaf or hard of hearing: a scoping review. Journal of Telemedicine and Telecare, 25(4), 249-260. https://doi.org/10.1177\%2F1357633X18755883

McCarthy, M., Leigh, G. y Arthur-Kelly, M. (2020a). Comparison of caregiver engagement in telepractice and in-person Family-Centered Early Intervention. The Journal of Deaf Studies and Deaf Education, 25(1), 33-42. https://doi.org/10.1093/deafed/enz037 
ATENCIÓN TEMPRANA EN TIEMPOS DE COVID-I 9: INVESTIGAR LA/S REALIDAD/ES DE LA TELEINTERVENCIÓN EN LAS PRÁCTICAS CENTRADAS EN LA FAMILIA

L. RODRÍGUEZ, I. HERRÁN, P. DE LA MANO, C. DÍAZ Y J. MARTíneZ

McCarthy, M., Leigh, G. y Arthur-Kelly, M. (2020b). Practitioners' self-assessment of Family-Centered Practice in telepractice versus in-Person Early Intervention. The Journal of Deaf Studies and Deaf Education, 26(1), 46-57. https://doi.org/10.1093/deafed/enaa028

McWilliam, R. A. (2000). Early Intervention in natural environments: a Five-Component Model. Division of Child Development, Department of Pediatrics, Vanderbilt University Medical Center. Recuperado de: http://www.kdec.org/HTML/2013Conference/EINE_ Five_Component_Model.pdf

McWilliam, R. A. (2010). Routines-based Early Intervention: supporting young children and their families. Paul H. Brookes.

McWilliam, R. A. (2016). Birth to three: Early Intervention. En Handbook of early childhood special education (pp. 75-88). Springer, Cham.

Melvin, K., Meyer, C. y Scarinci, N. (2020). What does a family who is "engaged” in early intervention look like? Perspectives of Australian speech-language pathologists. International Journal of Speech-Language Pathology, 1-11. https://doi.org/10.1080/17549507.202 0.1784279

Molini-Avejonas, D., Rondon-Melo, S., De la Higuera Amato, C. A. y SameLLI, A. G. (2015). A systematic review of the use of telehealth in speech, language and hearing sciences. Journal of Telemedicine and Telecare, 21(7), 367-376. https://doi. org/10.1177\%2F1357633X15583215

Moore, T. G. (2012). Rethinking early childhood intervention services: Implications for policy and practice. Conference and 1st Asia-Pacific Early Childhood Intervention Conference 2012, 9th August, Perth, Western Australia.

Moretti, F., Van Vliet, L., Bensing, J., Deledda, G., Mazzi, M., Rimondini, M., Zimmermann, Ch. y Fletcher, I. (2011). A standardized approach to qualitative content analysis of focus group discussions from different countries. Patient Education and Counseling, 82(3), 420-428. https://doi.org/10.1016/j.pec.2011.01.005

Navas, P., Verdugo, M. Á., Torres, S. M., Sainz, F. y Hernández, A. A. (2017). Derechos y calidad de vida en personas con discapacidad intelectual y mayores necesidades de apoyo. Siglo Cero, 48(4), 7-66. https://dx.doi.org/10.14201/scero2017484766

Neely, L., Rispoli, M., Gerow, S., Hong, E. R. y Hagan-Burke, S. (2017). Fidelity outcomes for autism-focused interventionists coached via telepractice: a systematic literature review. Journal of Developmental and Physical Disabilities, 29(6), 849-874. https://doi. org/10.1007/s10882-017-9550-4

Nind, M., Flewitt, R. y Payler, J. (2010). The social experience of early childhood for children with learning disabilities: inclusion, competence and agency. British Journal of Sociology of Education, 31(6), 653-670. https://doi.org/10.1080/01425692.2010.515113

O'Leary, Z. (2004). The essential guide to doing research. Sage Publications.

Olsen, S., Fiechtl, B. y Rule, S. (2012). An evaluation of virtual home visits in Early Intervention: feasibility of "Virtual Intervention". Volta Review, 112(3), 267-281. https:// doi:10.17955/tvr.112.3.m.702

Organización de Naciones Unidas. (2006). Convención Internacional sobre los Derechos de las Personas con Discapacidad. Adoptada por la Asamblea General de las Naciones Unidas el 13 de diciembre de 2006, durante el sexagésimo primer periodo de sesiones de la Asamblea General, por la Resolución 61/106. Recuperado de http://www.oas.org/DIL/ esp/A-RES_61-106_spa.pdf

Organización Mundial de la Salud. (?2001)? Clasificación Internacional del Funcionamiento de la Discapacidad y de la Salud. Ginebra.

Ediciones Universidad de Salamanca / CC BY-NC-ND

Siglo Cero, vol. 52, número extraordinario, 2021, pp. 75-117 
ATENCIÓN TEMPRANA EN TIEMPOS DE COVID-I 9: INVESTIGAR LA/S REALIDAD/ES DE LA TELEINTERVENCIÓN EN LAS PRÁCTICAS CENTRADAS EN LA FAMILIA L. RODRÍGUEZ, I. HERRÁN, P. DE LA MANO, C. DÍAZ Y J. MARTínez

Organización Mundial de la Salud. (2020). Declaración de pandemia mundial. Recuperado de https://www.who.int/es/news/item/27-04-2020-who-timeline---covid-19

O’Toole, C., Lyons, R., Ó’Doibhlin, D., O’Farrell, F. y Houghton, C. (2020). Stage 1 Registered Report: the experiences and perceptions of parent-child interaction therapy for parents of young children with communication difficulties: a qualitative evidence synthesis protocol (version 2). HRB Open Research, 2(36), 36. https://doi.org/10.12688/hrbopenres.12974.2

Owens, D. y Batchelor, C. (1996). Patient satisfaction and the elderly. Social Science and Medicine, 42(11), 1483-1491. https://doi.org/10.1016/0277-9536(95)00234-0

Paul, R. (2001). Language disorders from infancy through adolescence (2. ${ }^{a}$ ed.). London Mosby.

Payne, G. y Payne, J. (2004). Key concepts in social research. Sage Publications.

Persky, S. (2011). Employing immersive virtual environments for innovative experiments in health care communication. Patient Education and Counseling, 82(3), 313-317. https://doi. org/10.1016/j.pec.2010.12.007

Poole, M. E., Fettig, A., McKee, R. A. y Gauvreau, A. N. (2020). Inside the virtual visit: using tele-intervention to support families in Early Intervention. Young Exceptional Children. https://doi.org/10.1177/1096250620948061

Preeti, K., Srinath, S., Seshadri, S. P., Girimaji, S. C. y Kommu, J. V. S. (2017). Lost timeneed for more awareness in early intervention of autism spectrum disorder. Asian Journal of Psychiatry, 25, 13-15. https://doi.org/10.1016/j.ajp.2016.07.021

Puchta, C. y Potter, J. (2004). Focus group practice. Sage.

Raskind, I. G., Shelton, R. C., Comeau, D. L., Cooper, H. L., Griffith, D. M. y KeGLER, M. C. (2019). A review of qualitative data analysis practices in health education and health behaviour research. Health Education and Behaviour, 46(1), 32-39. https://doi. org/10.1177\%2F1090198118795019

Raver, S. A. y Childress, D. C. (2015). Family-centered early intervention: supporting infants and toddlers in natural environments. Brookes.

Real Decreto 463/2020, de 14 de marzo, por el que se declara el estado de alarma para la gestión de la situación de crisis sanitaria ocasionada por el COVID-19. (2020). Boletín Oficial del Estado, 67, sección I, de 14 de marzo de 2020, 25390 a 25400. Recuperado de https://www. boe.es/eli/es/rd/2020/03/14/463/con

Royal College of Speech and Language Therapists. (2006). Communicating Quality 3: $R C S L T$ 's guidance on best practice in service organisation and provision. Scotprint.

Sameroff, A. (2009). The Transactional Model of Development: how children and contexts shape each other. American Psychological Association.

Shonkoff, J. P. (2010). Building a new biodevelopmental framework to guide the future of early childhood policy. Child Development, 81(1), 357-367. https://doi.org/10.1111/ j.1467-8624.2009.01399.x

Silverman, D. (2005). Doing qualitative research (2. ${ }^{\text {a }}$ ed.). Sage Publications.

Sutherland, R., Trembath, D. y Roberts, J. (2018). Telehealth and autism: a systematic search and review of the literature. International Journal of Speech-Language Pathology, 20(3), 324-336. https://doi.org/10.1080/17549507.2018.1465123

TAMARIT, J. (2015). La transformación de los servicios hacia la calidad de vida. Una iniciativa de innovación social de FEAPS. Siglo Cero, 46(3), 47-71. https://dx.doi.org/10.14201/scero20154634771 
Trivette, C. M. y Dunst, C. J. (2000). Recommended practices in family-based practices. En S. Sandall, M. McLean y B. J. Smith (Eds.), DEC Recommended Practices in Early Intervention/Early Childhood Special Education (pp. 39-46). Sopris West.

Tucker, J. K. (2012). Perspectives of speech-language pathologists on the use of telepractice in schools: the qualitative view. International Journal of Telerebabilitation, 4(2), 47-59. https://doi.org/10.5195/ijt.2012.6102

Turnbull, A. P., Brown, I. y Turnbull, H. R. (2004). Families and people with mental retardation and quality of life: international perspectives. AAMR.

Valverde, B. B. D. R. y Jurdi, A. P. S. (2020). Analysis of the relationship between Early Intervention and family quality of life. Revista Brasileira de Educação Especial, 26(2), 283-298.

Vilaseca, R. M., Galván-Bovaira, M. J., González-del-Yerro, A., Baqués, N., OliveiRA, C., Simó-Pinatella, D. y Giné, C. (2019). Training needs of professionals and the family-centered approach in Spain. Journal of Early Intervention, 41(2), 87-104. https:// doi.org/10.1177\%2F1053815118810236

Watts, N., McLeod, S. y McAllister, L. (2009). Speech language pathologists' and other allied health professionals' perceptions of working with parents' families. En N. WATTS y S. McLeOd (Eds.), Working with families in speech language pathology (pp. 39-71). Plural Publishing.

Westby, C. E. (1990). Ethnographic interviewing: asking the right questions to the right people in the right ways. Journal of Childhood Communication Disorders, 13(1), 101-111. https:// doi.org/10.1177\%2F152574019001300111

Westby, C., Burda, A. y Mehta, Z. (2003). Asking the right questions in the right ways: strategies for ethnographic interviewing. The ASHA Leader, 8(8), 4-17. https://leader.pubs. asha.org/doi/full/10.1044/leader.FTR3.08082003.4 


\begin{tabular}{|l|l|l|}
\hline \multicolumn{2}{|c|}{$\begin{array}{c}\text { ApÉNDICE 1. Entrevista a las familias utilizada en el estudio. Adaptación a partir } \\
\text { de las 9 }\end{array}$} \\
\hline Dimensiones Sociales en la Entrevista Etnográfica (Westby, 1990)
\end{tabular}




\begin{tabular}{|c|c|c|c|c|}
\hline \multicolumn{5}{|c|}{$\begin{array}{l}\text { ApÉNDICE 1. Entrevista a las familias utilizada en el estudio. Adaptación a partir de } \\
\text { las } 9 \text { Dimensiones Sociales en la Entrevista Etnográfica (Westby, 1990) (cont.) }\end{array}$} \\
\hline Dimensión & $\begin{array}{l}\text { Subcategorías } \\
\text { dimensionales } \\
\text { (en su caso) }\end{array}$ & \multicolumn{3}{|c|}{ Ejemplos / tipo de pregunta (para unificar) } \\
\hline $\begin{array}{l}\text { D2. } \\
\text { Actores/ } \\
\text { protago- } \\
\text { nistas }\end{array}$ & \multicolumn{4}{|c|}{$\begin{array}{l}\text { Convivencia/composición familiar. } \\
\text { Personas que habéis convivido. } \\
\text { Edad de las personas. } \\
\text { Composición familiar. }\end{array}$} \\
\hline $\begin{array}{l}\text { D3. } \\
\text { Acciones/ } \\
\text { necesidades } \\
\text { de apoyo }\end{array}$ & \multicolumn{4}{|c|}{$\begin{array}{l}\text { Define cuáles son las necesidades de apoyo de tu hijo, si ha presentado } \\
\text { tu hijo dificultades en la conducta (seguimiento de rutinas, transiciones, } \\
\text { sueño, alimentación, etc.). }\end{array}$} \\
\hline $\begin{array}{l}\text { D4. } \\
\text { Activida- } \\
\text { des/rutinas }\end{array}$ & \multicolumn{4}{|c|}{$\begin{array}{l}\text { Describe un día tipo en la teleintervención: ej., si ocurría un lunes, qué } \\
\text { pasaba ese día y en qué momento encajaban la teleintervención. } \\
\text { Otros tipos de comunicación con el profesional de referencia: ¿envío de } \\
\text { vídeos, llamadas o mensajes? }\end{array}$} \\
\hline $\begin{array}{l}\text { D6. } \\
\text { Reflexión }\end{array}$ & \multicolumn{4}{|c|}{$\begin{array}{l}\text { Qué fortalezas/competencias/aprendizajes han surgido de vuestra fami- } \\
\text { lia a raíz de esta experiencia. Cómo ha influido la teleintervención en la } \\
\text { relación/colaboración con los profesionales. } \\
\text { Relación con el profesional: con cuántos profesionales has tenido rela- } \\
\text { ción. Valoración de la relación. }\end{array}$} \\
\hline $\begin{array}{l}\text { D7. } \\
\text { Tiempo/s }\end{array}$ & \multicolumn{4}{|c|}{$\begin{array}{l}\text { Cómo es esa visita, qué pasa ahí y qué pasa entre semana. } \\
\text { ¿Cómo has percibido tú todo lo que ha pasado entre visita y visita? }\end{array}$} \\
\hline $\begin{array}{l}\text { D8. } \\
\text { Objetivo }\end{array}$ & \multicolumn{4}{|c|}{$\begin{array}{l}\text { Indagar dónde está el foco. A qué cosas has renunciado en el confina- } \\
\text { miento, en cuanto a tu rol parental. Cambio de prioridades. Replantea- } \\
\text { miento de vuestras prioridades, de dónde ha surgido esa necesidad, ese } \\
\text { motor de cambio. } \\
\text { Sensación de competencia de la familia/confianza. } \\
\text { Apoyos solicitados. Tipos de apoyo que se han prestado. } \\
\text { Qué cosas hemos aprendido que puedan mejorar las futuras intervenciones. }\end{array}$} \\
\hline $\begin{array}{l}\text { D9. } \\
\text { Emociones }\end{array}$ & $\begin{array}{l}\text { Las emociones } \\
\text { vividas y } \\
\text { expresadas. }\end{array}$ & $\begin{array}{l}\text { Preocupación, } \\
\text { miedo, } \\
\text { disfrute... }\end{array}$ & $\begin{array}{l}\text { E mociones } \\
\text { que han surgi- } \\
\text { do durante el } \\
\text { proceso. } \\
\text { La construc- } \\
\text { ción de tu pro- } \\
\text { pia narrativa. }\end{array}$ & $\begin{array}{l}\text { Estas son las cosas } \\
\text { que te han pasado, } \\
\text { pero ¿cómo lo has } \\
\text { vivido?, ¿cómo lo es- } \\
\text { tás narrando? } \\
\text { ¿Cómo crees que lo } \\
\text { han vivido los profe- } \\
\text { sionales?, ¿qué les pre- } \\
\text { guntarías a ellos si tu- } \\
\text { vieras la oportunidad? }\end{array}$ \\
\hline
\end{tabular}




\begin{tabular}{|c|c|c|c|c|}
\hline \multicolumn{5}{|c|}{$\begin{array}{l}\text { ApÉNDICE 1. Entrevista a las familias utilizada en el estudio. Adaptación a partir } \\
\text { de las } 9 \text { Dimensiones Sociales en la Entrevista Etnográfica (Westby, 1990) (cont.) }\end{array}$} \\
\hline Dimensión & $\begin{array}{l}\text { Subcategorías } \\
\text { dimensionales }\end{array}$ & \multicolumn{3}{|c|}{ Ejemplos / tipo de pregunta (para unificar) } \\
\hline \multirow[t]{2}{*}{$\begin{array}{l}\text { Pregunta } \\
\text { de cierre/ } \\
\text { conclusivas }\end{array}$} & \multirow[t]{2}{*}{$\begin{array}{l}\text { En sus pro- } \\
\text { pias palabras. }\end{array}$} & \multirow[t]{2}{*}{$\begin{array}{l}\text { Tratan de cla- } \\
\text { rificar lo que } \\
\text { están contan- } \\
\text { do las familias. }\end{array}$} & $\begin{array}{l}\text { Proponer que } \\
\text { lo narre para } \\
\text { que una per- } \\
\text { sona ajena lo } \\
\text { entienda. }\end{array}$ & $\begin{array}{l}\text { Hipotéticas: imagi- } \\
\text { na que un fontane- } \\
\text { ro, por ejemplo, es- } \\
\text { tuviera ahora aquí, } \\
\text { explícale a esa per- } \\
\text { sona cuál es la situa- } \\
\text { ción que has vivido, } \\
\text { qué ha pasado, qué } \\
\text { es la teleinterven- } \\
\text { ción en Atención } \\
\text { Temprana. }\end{array}$ \\
\hline & & & $\begin{array}{l}\text { Visibilizar y } \\
\text { evidenciar lo } \\
\text { sustancial, el } \\
\text { cierre que sea } \\
\text { conclusivo del } \\
\text { proceso lleva- } \\
\text { do a cabo. }\end{array}$ & $\begin{array}{l}\text { Me puedes comen- } \\
\text { tar una de las cues- } \\
\text { tiones/situaciones } \\
\text { en las que te has sen- } \\
\text { tido especialmente } \\
\text { apoyado y esto haya } \\
\text { mejorado la calidad } \\
\text { de vida durante ese } \\
\text { tiempo. } \\
\text { La de mayor huella } \\
\text { y si se te ocurre al- } \\
\text { guna otra más. } \\
\text { Ponnos un ejemplo } \\
\text { de una situación/ } \\
\text { cuestiones en las } \\
\text { que os hayáis vis- } \\
\text { to especialmente } \\
\text { apoyados, algo que } \\
\text { os haya aliviado y } \\
\text { que haya mejorado } \\
\text { vuestra calidad de } \\
\text { vida familiar. }\end{array}$ \\
\hline
\end{tabular}




\begin{tabular}{|c|c|c|}
\hline \multicolumn{3}{|r|}{$\begin{array}{l}\text { iilias utilizada en el estudio. Adaptación a partir } \\
\text { la Entrevista Etnográfica (Westby, 1990) (cont.) }\end{array}$} \\
\hline Dimensión & $\begin{array}{c}\text { Subcategorías } \\
\text { dimensionales } \\
\text { (en su caso) }\end{array}$ & Ejemplos / tipo de pregunta (para unificar) \\
\hline Despedida & \multicolumn{2}{|c|}{$\begin{array}{l}\text { Con esto terminaríamos la sesión. Hacerle saber que la entrevista ha } \\
\text { terminado: poner en palabras que la grabación también finaliza. } \\
\text { Te agradecemos todas las aportaciones que has hecho, porque son } \\
\text { muy valiosas para ayudarnos a comprender cómo se han desarrollado } \\
\text { las prácticas de AT mediante teleintervención. } \\
\text { Este estudio servirá para inspirar futuros trabajos: investigación, do- } \\
\text { cencia, profesionales, Administración. } \\
\text { Quedamos a tu disposición para todo lo que puedas necesitar. Por } \\
\text { medio de la coordinadora o directamente si te surgen dudas, revo- } \\
\text { car algo que hayas dicho, matizar... Te vamos a seguir "escuchando" } \\
\text { aunque hayamos terminado la entrevista. } \\
\text { Si quieres añadir algo más: correo-e o teléfono con el que ya hemos } \\
\text { contactado previamente (la persona que ha hecho esa entrevista } \\
\text { determinada). } \\
\text { Agradecimiento y avanzar que vamos a cerrar sesión y conexión, para } \\
\text { gue no se asuste si desaparecemos. }\end{array}$} \\
\hline
\end{tabular}




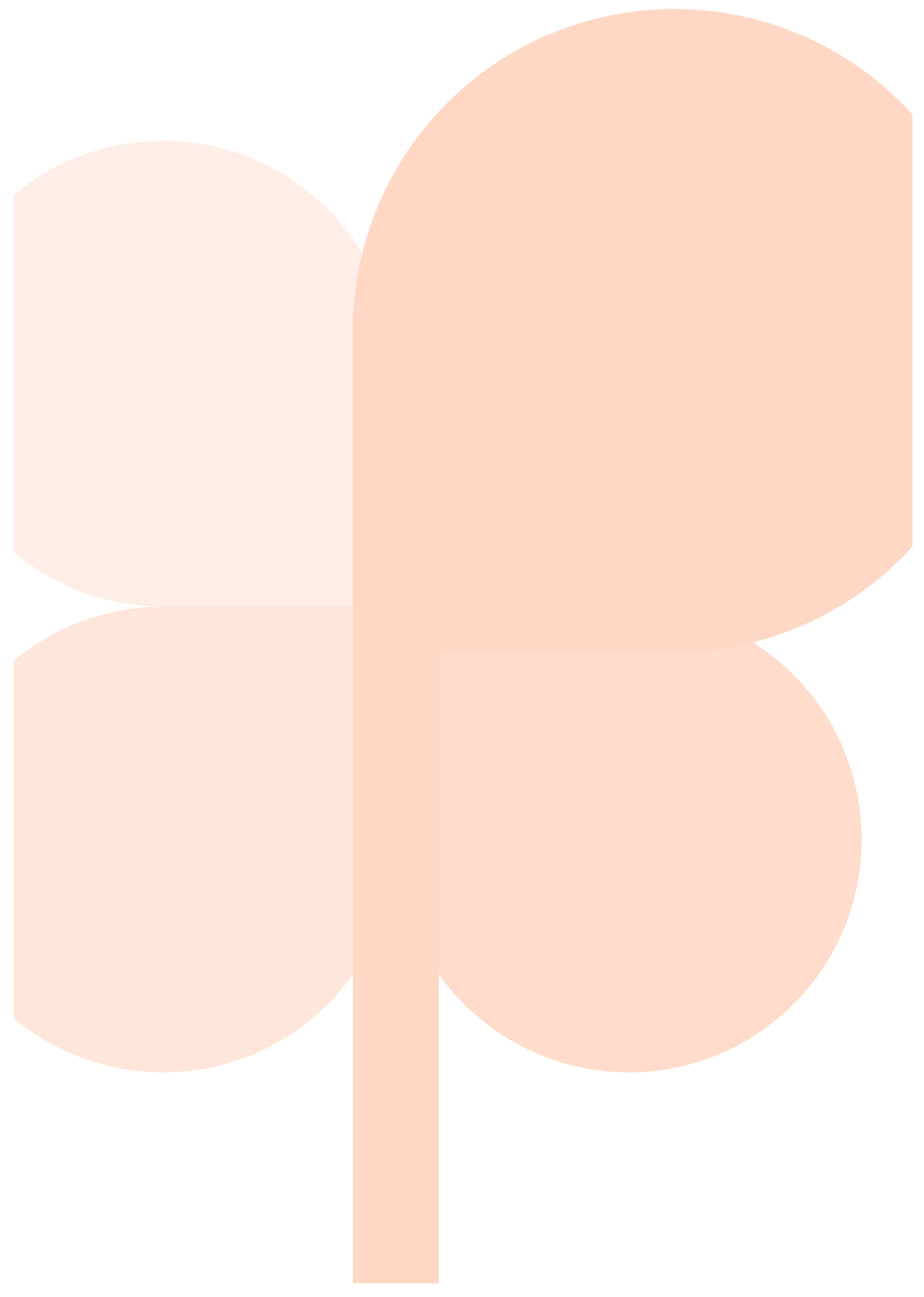

